\title{
Modeling the temporal evolution of Cocoa black pod rot disease caused by Phytophthora megakarya*
}

\author{
C. Nembot ${ }^{a}$, P. Takam Soh ${ }^{b}$, G. M. ten Hoopen ${ }^{c, d}$, Y. Dumonte,f,g \\ ${ }^{a}$ University of Yaounde I, Department of Plant Biology, Yaounde, Cameroon, \\ ${ }^{b}$ University of Yaounde I, Department of Mathematics, Yaounde, Cameroon \\ ${ }^{c}$ CIRAD, UPR Bioagresseurs, CRC, Trinidad and Tobago \\ ${ }^{d}$ Bioagresseurs, Univ Montpellier, CIRAD, Montpellier, France \\ ${ }^{e}$ CIRAD, Umr AMAP, Pretoria, South Africa, \\ $f$ AMAP, Univ Montpellier, CIRAD, CNRS, INRA, IRD, Montpellier, France, \\ ${ }^{g}$ University of Pretoria, Department of Mathematics and Applied Mathematics, Pretoria, South Africa
}

October 26, 2018

\begin{abstract}
Black pod rot, caused by Phytophthora megakarya, is the main cause of cocoa losses in Cameroon. A few studies have focused on describing black pod epidemics in cocoa yet numerous questions remain. Here, an epidemiological model describing the temporal evolution of cocoa black pod, taking into account the development stages of pods, is developed and studied. In particular, the relative importance of primary and secondary infection in disease dynamics is investigated. Our theoretical study shows the existence of a disease free equilibrium and at at least one endemic equilibrium. We highlight two threshold parameters, related to direct and indirect infections that summarize all possible dynamics of the system. Then, based on the literature, we define a periodic pod recruitment function, and provide several numerical simulations to study the impact of phytosanitary pod removal on disease dynamics. We show that intense and regular sanitary harvest could lead to complete disease eradication. Our results also highlight the importance of the environmental spores reservoir in disease dynamics, such that future field experiments and observations should focus on it.
\end{abstract}

Keywords:Cocoa, black pod rot, disease, epidemiological model, analysis, simulations.

\section{Introduction}

Cocoa (Theobroma cacao L.) is one of the most important perennial crops worldwide, with an estimated world production of 4.25 million tons in 2014/15 with an estimated value of around 12.6 billion US dollars [23] (ICCO, 2017). However, between 20 to $30 \%$ of annual yield is lost due to pests and diseases. Some even estimate annual yield losses to be as high as $40 \%$ [32]. At a global scale, the most important disease is cocoa black pod rot, which is due to several species of Phytophthora. The most important species globally is P. palmivora [13], since it is pan-tropical, occurring in all cacao producing countries. However, the most aggressive and damaging species is P. megakarya (C.M. Brasier \& M.J. Griffin) ([32],[2] and [3]). Phytophthora megakarya is only

\footnotetext{
*corresponding author: Yves Dumont. email: yves.dumont@cirad.fr
} 
found in countries in West and Central Africa [3]. In both Nigeria and Cameroon, $P$ megakarya has replaced $P$. palmivora as a significant causal agent of black pod rot and is currently in an invasive phase in Ghana and Ivory Coast [5]. Yet, since these West and Central African countries produce around $70 \%$ of all cocoa beans worldwide. In Cameroon, 5th cocoa producer in the world in 2014/15, cocoa black pod rot is only due to P. megakarya and losses often reach up to $80-90 \%$ when plantations are poorly managed $[21,31]$.

It is known that cocoa production in Cameroon, as elsewhere, is tightly linked to climate, especially rainfall. In Cameroon, flowering of the cacao tree is induced by the first rains after a dry season. Flowering typically lasts for about 1 to 2 months and it takes around 5-6 months from pollination to full pod ripening. According to their growing stages, developing pods are subdivided into cherelles, young, mature, and ripe pods. Developing pods are called cherelles until the stage at which no further cherelle wilting, a cocoa physiological fruit thinning mechanism, occurs. When rainfall is evenly distributed throughout the year, crop production can be spread out over many months. In contrast, when rainfall distribution is more unevenly spread throughout the year, like in Cameroon, cropping (production) is generally seasonal. In Cameroon, two types of rainfall areas exists where cocoa is cropped, a unimodal rainfall area, with one dry and one wet season and a bimodal rainfall area, with one long and one short dry season and also one short and one long rainy season. The short and long rainy season in bimodal areas are usually from March/April to June/July and August to November, respectively. The principal flowering period coincides with the first rainy season. The short dry spell in bimodal rainfall areas can hamper the development of Phytophthora pod rot.

Phytophthora megakarya: life cycle and epidemiology There are two means of infection by $P$. megakarya, direct transmission (pod to pod infection) and indirect transmission (from an environmental reservoir to a healthy pod). P. megakarya has 4 invective stages, the first one is the mycelial stage and the others are the three spore types, sporangia, zoospores and chlamydospores. All four stages can directly or indirectly cause infection. Primary inoculum, which survives in the soil in the form of chlamydospores or mycelium during the dry season and/or when pods are absent, is activated under wet and humid conditions and develops sprorangia. Through rain-splash or by certain insects such as ants, sporangia or the motile biflagellate zoospores they contain, are transported onto pods where they can establish an infection [27]. Two factors that greatly contribute to the success of P. megakarya as cocoa pathogen is its ability to rapidly form appressoria (a flattened and thickened tip of a hyphal branch, that facilitates penetration of the host plant), in much greater quantities than P. palmivora [3] as well as its capability to shed zoospores earlier and twice as much than P. palmivora [11]. Successful infection then results in the generation of secondary inoculum which gets dispersed again primarily through rain-splash, causing secondary infections. Black pod disease incidence in the field is influenced by different environmental conditions yet several studies have established rain as a primary factor governing epidemics $([11],[16])$.

Moreover, under humid conditions a single pod may produce up to 4 million sporangia (each containing up to around 40 motile zoospores), that are mainly disseminated by rain splash [11]. According to [11] P. megakarya inoculum can survive up to 18 months in the soil of cocoa plantations. An overview of the life cycle of Phytophthora megakarya is presented in Fig. 1, page 3.

Although P. megakarya can infect cocoa pods at all developmental stages, studies from Cameroon 


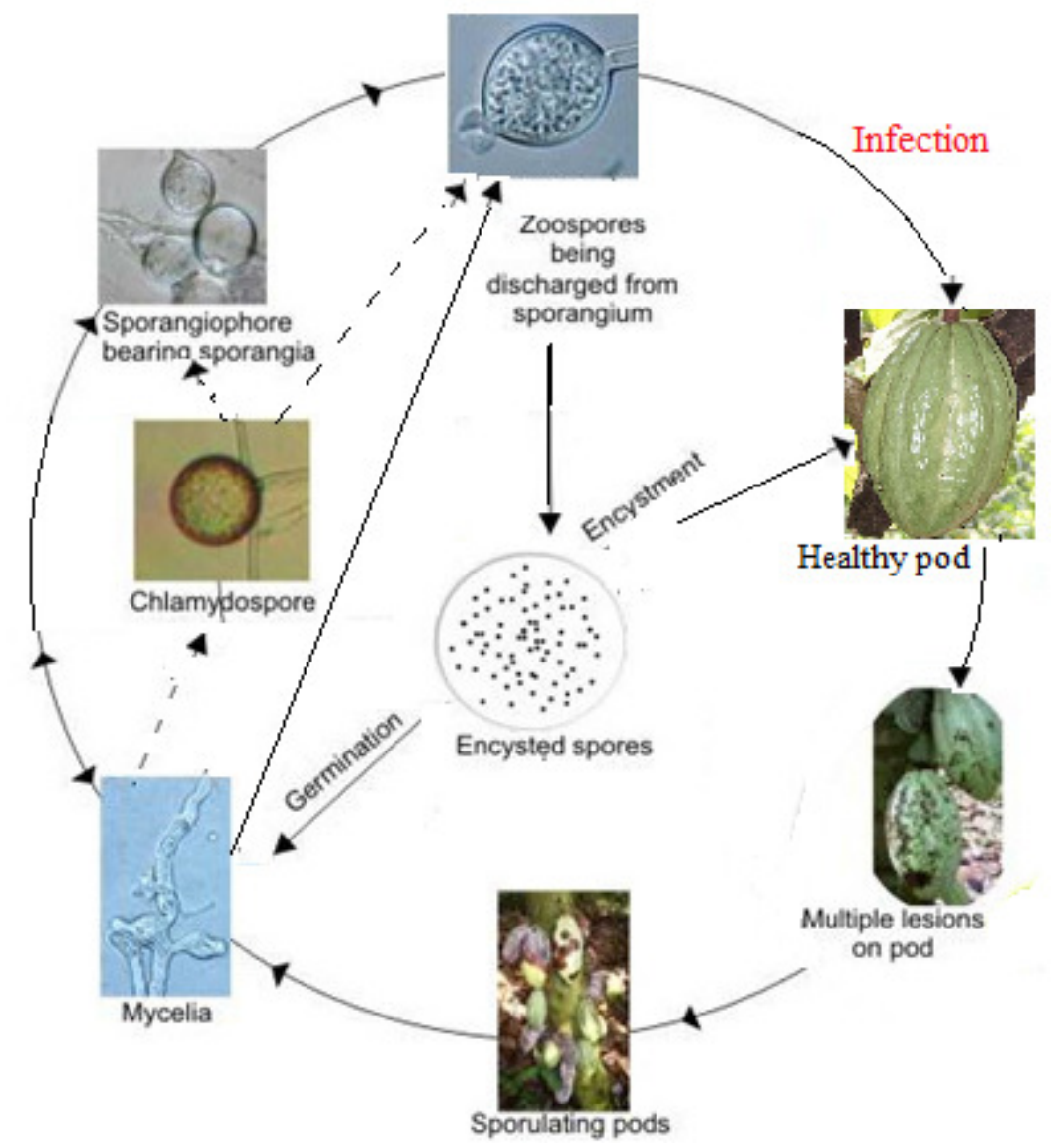

Figure 1: The life cycle of Phytophthora megakarya highlighting the main spores type and describing infection process on cocoa pod

(modified from [1]).

showed that susceptibility and the risk of attack depends among others on the developmental stage of cocoa pods [18, 35]. The growth cycle of a cocoa pod can be divided into three phases: an early accelerating/cell division phase, a linear/cell enlargement phase and a saturation phase for ripening/maturation ([20]; see Fig. 2, page 4). This growth pattern of cocoa pods generally follows a sigmoid curve ([36]; Fig. 2). Based on the literature $[18,35]$ it is assumed that the first growth stage called cherelles is when pods are most susceptible. Ripe pods, even though at the end of the production cycle they can still become infected, likely contribute little to Phytophthora pod rot epidemics, even though ripe pods can still become infected. This is because ripe infected pods are often and regularly being harvested. The full grown beans, although of less quality in the case of infection, are still of economic interest to the farmer.

Control of cocoa black pod disease Cocoa black pod is generally controlled through fungicide applications, combined with the use of partially resistant or tolerant cocoa cultivars and appropriate cultural practices, such as phytosanitation [30]. Biological control seems to be promising but no commercial products are as yet available [29]. An increase in the effectiveness of control can be expected when these control methods are rationally combined [9]. Under field conditions, the natural activity of antagonistic and hyperparasitic microbes can also contribute to a decay of 


\section{Fruit growth curve}

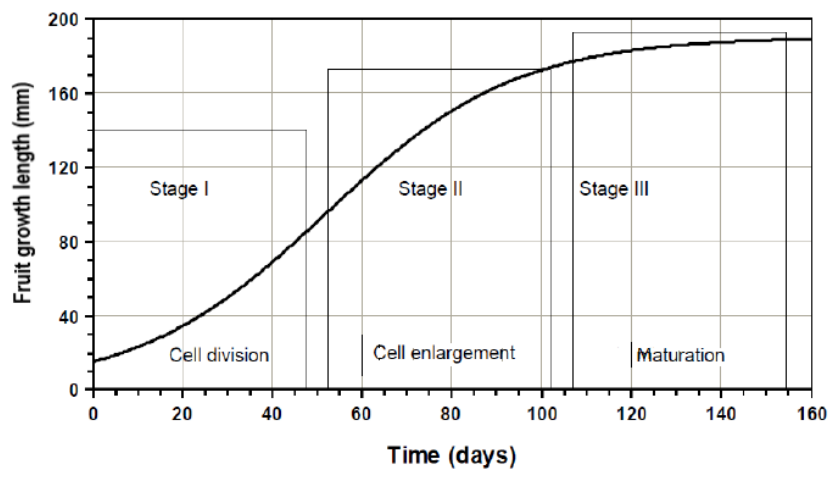

Figure 2: Generalised fruit development stages and Sigmoid growth curve with specific cacao pod development periods

(modified from [36]).

Phytophtora sp. viability [26]. Phytosanitary pod removal is a preventive method which consists of cleaning trees at the beginning of the season by removing mummified fruits left from the previous season, and the subsequent regular removal of diseased pods which are the source of secondary inoculum [30]. However, phytosanitation is time consuming and relatively difficult to put in place. Fungicide applications are however, effective, especially economically speaking [19]. Metalaxyl is the main active ingredient of the most effective fungicides for preventing/controlling black pod disease due to $P$. megakarya. Yet, fungicide use has numerous negative externalities, such as human health problems, pollution, reductions in the populations of beneficial organisms, and emergence of secondary diseases or pathogen resistance to pesticides [4]. Thus, there are compelling reasons to come up with alternative, innovative and more effective control strategies. However, to do so we need to identify control levers through which it is possible to exert control. This can only be done through a better understanding of the disease dynamics. If Field expertise and experiments are of utmost importance for this understanding, Modeling can also be a very helpful approach, in particular when experiments are missing and/or difficult to conduct or to test new assumptions. However, relatively few studies have focused on understanding the temporal evolution of $P$. megakarya epidemics, and there are still many questions regarding the factors that govern these dynamics. For instance, the relative importance of the two different sources of inoculum (primary and secondary) and spore dispersal dynamics are not well understood. We believe that Modeling can be an effective approach to provide answers to these questions and help to improve or build efficient control strategies, and, also, to better focus on future field experiments.

That is why the aims of this paper are threefolds: first, to understand the dynamics of the diseases in the case of a constant or periodic pods production; second, to understand what are the main parameters that drive these dynamics; and, third, to investigate the impact of phytosanitation (a cultural control strategy).

The paper is structured as follows. In section 2, we first describe the Phytophthora model. Then, in section 3, we provide a theoretical analysis of the model, followed by numerical simulations in section 4, where we investigate the impact of phytosanitary pod removal on disease dynamic and compare different scenarii of control strategies. Finally, in Section 5, we conclude and discuss possible perspectives and extensions. 


\section{The Phytophthora model}

Our model classifies hosts (Cocoa pods) in two epidemiological states: susceptible $(S)$ and infectious $(I)$. Transmissions from pod to pod and from the environment to pod, through spores, are the only ways a black pod rot epidemic can expand. Healthy (susceptible) pods are classified in three classes according to their developmental stage: the first developmental stage of cocoa pods, called Cherelles $\left(S_{1}\right)$, then young and mature pods $\left(S_{2}\right)$, and then, ripe pods $\left(S_{3}\right)$, that can be harvested and provide the beans used for commercialization. Based on our knowledge of the pathosystem, the infectious pods compartment $(I)$ has two sub-compartments according to the ways of transmission: spores produced by infected pods and released in the environment, responsible for secondary infections, $\left(S_{p, e}\right)$, and spores produced by infected pods and directly responsible for primary infections $\left(S_{p, i}\right)$. Our epidemiological system is represented in Fig. 3, page 5.

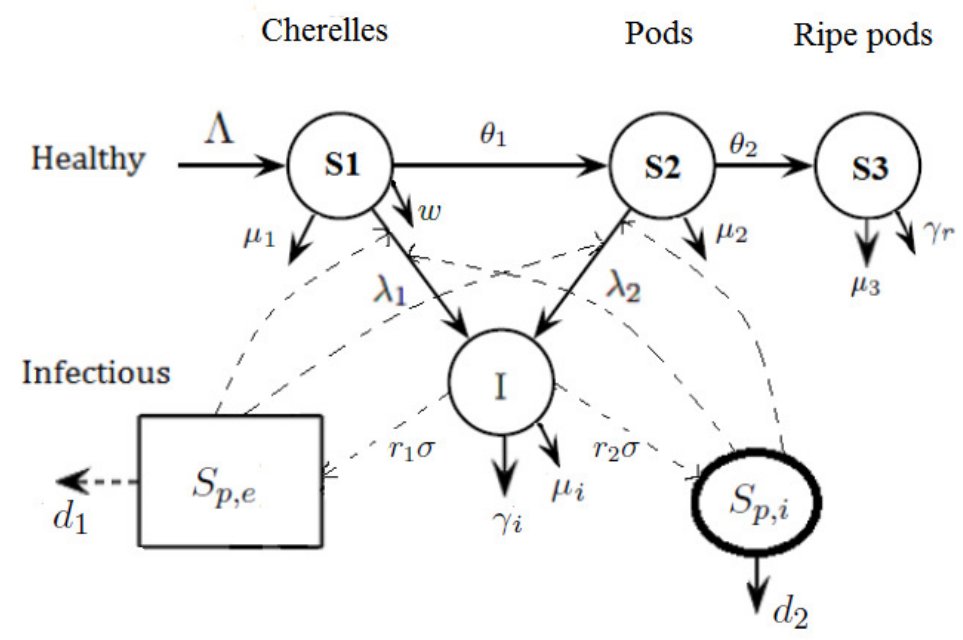

Figure 3: A compartmental model of the black pod rot epidemic

We only consider an inflow in the cherelle compartment of new susceptible hosts at rate $\Lambda$ : we first assume $\Lambda$ constant which corresponds to a regular production along the year in Central America, while periodic $\Lambda$ corresponds to region where the production is seasonal, like in Cameroon. The other parameters $\mu_{1}, w$, and $\theta_{1}$ represent respectively the natural death rate, the additional death rate due to wilt, and the maturation rate. Note that we distinguish $\omega$ from $\mu_{1}$, since cherelle wilt can vary greatly from plot to plot and/or from one year to the next. This is a natural process which plays the role of a regulation factor on each tree. At the pod stage, $\mu_{2}$ and $\theta_{2}$ represent respectively the mean death rate, and the maturation rate from immature pods to mature or ripe pods. $\mu_{3}$ is the mean death rate of ripe pods by excessive ripening or mummification, and $\gamma_{r}$ is the harvest rate of ripe pods. Finally, $\gamma_{I}$ represents the rate of phytosanitary pod removal in the plot and $\mu_{I}$ is the mean death rate of infected pods by mummification and saprophytic fungi which leads to the end of infectivity. At the spores stages, $\sigma$ is the production rate of spores by infected pods. $r_{2}$ and $r_{1}$ are respectively the releasing speed and the shedding rate of spores in the environment through infected plant tissue. $d_{2}$ and $d_{1}$ are respectively the inactivation speed of spores released by infected pods due to parasitism and senescence and the natural decay of spores in the environment (we assume for the spores compartment that $r_{1}+r_{2}<1$ ). According to the maturity stage $k$, where $k=1$ or 2 , susceptible pods, in contact with spores, coming either from 
the environment (primary inoculum), or spread directly from infected pods (secondary inoculum), are contaminated according to the following infection rates

$$
\lambda_{k}\left(S_{p, e}, S_{p, i}\right)=\beta_{1}^{k} \frac{S_{p, e}}{K_{1}+S_{p, e}}+\beta_{2}^{k} \frac{S_{p, i}}{K_{2}+S_{p, i}}, \quad k=1,2
$$

where $\beta_{1}^{k}$ and $\beta_{2}^{k}$ are respectively the (maximal) rate of primary and secondary infection. Indeed, we take into account that the total number of spores produced by infected pods in a plot is extremely large, such that a saturation effect occurs in the transmission rate. That is why Michaelis-Menten functionals have been considered. The parameters $K_{1}$ and $K_{2}$ in (1 page 6 , are called the Michaelis constants and represent the quantity of Phytophthora megakarya spores yielding $50 \%$ of chance for a pod to be infected by one specific inoculum. The latent time between the infection of pods by Phytophthora megakarya and the manifestation of firsts macroscopic symptoms being really short ( 3 -4 days[5]), there is often no way to distinguish an infected pod from an infectious one. This is why the delay has not been considered in the infection dynamic.

Given that $S_{3}$ does not play an important role in the infectious dynamic, the study of the system will be done without this compartment. Thus, we deduce that our system is governed by the following system of ODEs:

$$
\left\{\begin{aligned}
\frac{d S_{1}}{d t} & =\Lambda-\theta_{1} S_{1}-\left[\frac{\beta_{1}^{1} S_{p, e}}{K_{1}+S_{p, e}}+\frac{\beta_{2}^{1} S_{p, i}}{K_{2}+S_{p, i}}\right] S_{1}-\mu_{1} S_{1}-w S_{1} \\
\frac{d S_{2}}{d t} & =\theta_{1} S_{1}-\left[\frac{\beta_{1}^{2} S_{p, e}}{K_{1}+S_{p, e}}+\frac{\beta_{2}^{2} S_{p, i}}{K_{2}+S_{p, i}}\right] S_{2}-\mu_{2} S_{2}-\theta_{2} S_{2}, \\
\frac{d I}{d t} & =\left[\frac{\beta_{1}^{1} S_{p, e}}{K_{1}+S_{p, e}}+\frac{\beta_{2}^{1} S_{p, i}}{K_{2}+S_{p, i}}\right] S_{1}+\left[\frac{\beta_{1}^{2} S_{p, e}}{K_{1}+S_{p, e}}+\frac{\beta_{2}^{2} S_{p, i}}{K_{2}+S_{p, i}}\right] S_{2}-\gamma_{I} I-\mu_{I} I \\
\frac{d S_{p, i}}{d t} & =r_{2} \sigma I-d_{2} S_{p, i} \\
\frac{d S_{p, e}}{d t} & =r_{1} \sigma I-d_{1} S_{p, e} .
\end{aligned}\right.
$$

We complete system (2) with non negative initial conditions

$$
\left(S_{1}(0), S_{2}(0), I(0), S_{p, i}(0), S_{p, e}(0)\right) \geq 0 .
$$

Some parameters values have been deduced from the bibliography, but for most of the parameters, the values have been estimated. This is summarized in Table 1, page 33.

In the next section, we study the dynamics of system (2), with a particular focus on the DiseaseFree (DFE) and Endemic (EE) Equilibria, and the conditions to have existence and to reach these equilibria. To this end, we will estimate the Basic Reproduction Number and other thresholds in order to provide informations how model's parameters may impact the disease dynamic and, also, to study the efficiency of ongoing phytosanitary strategies (related to $\gamma$ ). 


\section{Mathematical analysis of the model}

System (2) can be rewritten in the following way

$$
\left\{\begin{array}{l}
\frac{d X}{d t}=A(X) X+F \\
X(0)=X_{0} \geq 0
\end{array}\right.
$$

with $X(t)=\left(S_{1}, S_{2}, I, S_{p, i}, S_{p, e}\right)^{T}$,

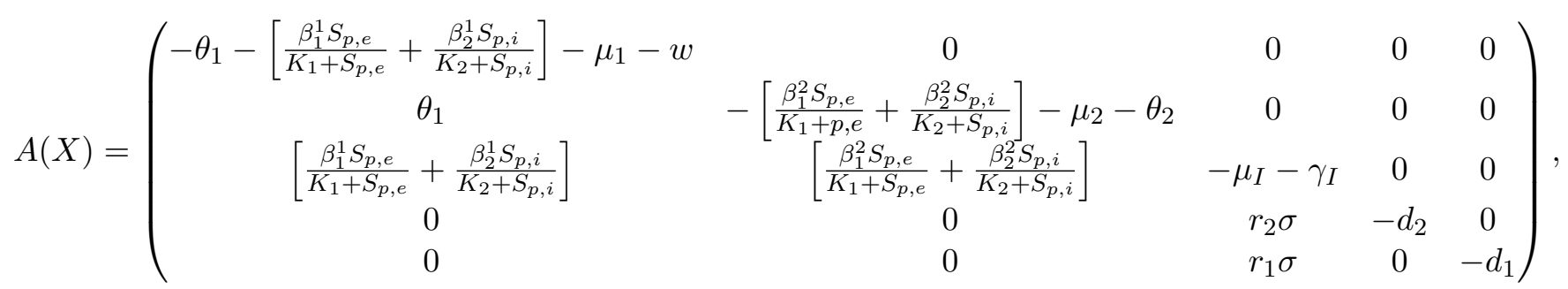

Note that $A(X)$ is a Metzler matrix, i.e a matrix such that off diagonal terms are non negative, for all $X \in \mathbb{R}_{+}^{5}$.

Thus, using the fact that $F \geq 0$, system (4) is positively invariant in $\mathbb{R}_{+}^{5}$, which means that any trajectory of the system starting from an initial state in the positive orthant $\mathbb{R}_{+}^{5}$ remains forever in $\mathbb{R}_{+}^{5}$.

Since the right hand side of system (2, page 6), is Lipschitz continuous, there exists an unique maximal solution. Using the "cascade method", we show that any compartment of (2) is bounded.

From $(2)_{1}$ (namely equation 1 in system (2)), we derive

$$
\frac{d S_{1}}{d t} \leq \Lambda-\theta_{1} S_{1}-\mu_{1} S_{1}-w S_{1}
$$

Setting

$$
S_{1}^{*}=\frac{\Lambda}{\mu_{1}+w+\theta_{1}},
$$

we deduce that $S_{1}(t) \leq S_{1}^{*}+\left(S_{1}(0)-S_{1}^{*}\right) * e^{-\left(\mu_{1}+w+\theta_{1}\right) t}$ such that, if $S_{1}(0)$ is chosen less than $S_{1}^{*}$, one has

$$
0 \leq S_{1}(t) \leq S_{1}^{*}
$$

Next, from $(2)_{2}$, and since $S_{1}(t) \leq S_{1}^{*}$, we derive

$$
\frac{d S_{2}}{d t} \leq \theta_{1} S_{1}-\left(\mu_{2}+\theta_{2}\right) S_{2} \leq \theta_{1} S_{1}^{*}-\left(\mu_{2}+\theta_{2}\right) S_{2}
$$

Then, setting

$$
S_{2}^{*}=\frac{\theta_{1} S_{1}^{*}}{\mu_{2}+\theta_{2}},
$$

we deduce that $S_{2}(t) \leq S_{2}^{*}+\left(S_{2}(0)-S_{2}^{*}\right) e^{-\left(\mu_{2}+\theta_{2}\right) t}$ such that, if $S_{2}(0)$ is chosen less than $S_{2}^{*}$, one has

$$
0 \leq S_{2}(t) \leq S_{2}^{*}
$$

Since we showed that all susceptible components, $S_{1,2}$, are bounded, we are able to show that the variable $I$ has an upper bound too. Thanks to the definition of "Michaelis-Menten" functions, we know that 


$$
\left\{\begin{array}{l}
\frac{S_{p, e}}{K_{1}+S_{p, e}} \leq 1 \\
\frac{S_{p, i}}{K_{2}+S_{p, i}} \leq 1
\end{array}\right.
$$

which implies

$$
\lambda_{i}\left(S_{p, e}, S_{p, i}\right)=\frac{\beta_{1}^{i} S_{p, e}}{K_{1}+S_{p, e}}+\frac{\beta_{2}^{i} S_{p, i}}{K_{2}+S_{p, i}} \leq \beta_{1}^{i}+\beta_{2}^{i} .
$$

Thus, setting

$$
I^{*}=\frac{\left(\beta_{1}^{1}+\beta_{2}^{1}\right) \mu_{2}\left(\mu_{1}+\theta_{1}\right)+\left(\beta_{1}^{2}+\beta_{2}^{2}\right) \theta_{1}}{\left(\gamma_{i}+\mu_{i}\right)\left(\mu_{2}+\theta_{2}\right)\left(\mu_{1}+\theta_{1}\right)} \Lambda,
$$

from $(2)_{4}$, we deduce that $I(t) \leq I^{*}+\left(I(0)-I^{*}\right) * e^{-\left(\gamma_{I}+\mu_{I}\right) t}$, such that if $I(0)$ is chosen smaller that $I^{*}$, one has $0 \leq I(t) \leq I^{*}$.

Finally, with the same reasoning, upper bounds for the spores compartments follow immediately:

$$
S_{p, i}(t) \leq \frac{r_{2} \sigma}{d_{2}} I^{*}
$$

and

$$
S_{p, e}(t) \leq \frac{r_{1} \sigma}{d_{1}} I^{*}
$$

According to the previous results, we can define the following positively invariant subset of $\mathbb{R}_{+}^{5}$

$$
\Omega=\left\{\left(S_{1}, S_{2}, I, S_{p, i}, S_{p, e}\right) \in \mathbb{R}_{+}^{5}: S_{1} \leq S_{1}^{*}, S_{2} \leq S_{2}^{*} ; I \leq I^{*}, S_{p, i} \leq \frac{r_{2} \sigma}{d_{2}} I^{*}, S_{p, e}(t) \leq \frac{r_{1} \sigma}{d_{1}} I^{*}\right\} .
$$

$\Omega$ is attractive, that is, solutions that start in $\Omega$ are bounded and remain in $\Omega$.

\subsection{Equilibria: existence, local, and global asymptotic stability}

In this section, we study the existence of equilibria (DFE and EE) related to system (2). We study the local and global asymptotic stability of DFE. We also compute the basic reproduction number $\mathcal{R}_{0}$ related to system (2). The equilibria of system (2) are obtained from the following equalities derived from (2) and representing the steady states of the system:

$$
\left\{\begin{aligned}
\left(\theta_{1}+\lambda_{1}\left(S_{p, e}, S_{p, i}\right)+\mu_{1}+w\right) S_{1} & =\Lambda \\
\left(\lambda_{2}\left(S_{p, e}, S_{p, i}\right)+\mu_{2}+\theta_{2}\right) S_{2} & =\theta_{1} S_{1} \\
\left(\gamma_{I}+\mu_{I}\right) I & =\lambda_{1}\left(S_{p, e}, S_{p, i}\right) S_{1}+\lambda_{2}\left(S_{p, e}, S_{p, i}\right) S_{2} \\
d_{2} S_{p, i} & =r_{2} \sigma I \\
d_{1} S_{p, e} & =r_{1} \sigma I
\end{aligned}\right.
$$

Setting $I=0$ in system (5), implies that $S_{p, i}, S_{p, e}$ and the infections forces $\lambda_{i}\left(S_{p, e}, S_{p, i}\right)$ are all equal to zero. Thus, we immediately deduce that

$$
\begin{cases}S_{1}^{d f e} & =\frac{1}{\theta_{1}+\mu_{1}+w} \Lambda \\ S_{2}^{d f e} & =\frac{\theta_{1}}{\left(\mu_{2}+\theta_{2}\right)\left(\theta_{1}+\mu_{1}+w\right)} \Lambda\end{cases}
$$

such that the DFE of system (2) is defined by

$$
D F E=\left(S_{1}^{d f e}, S_{2}^{d f e}, 0,0,0\right)^{T} .
$$




\subsubsection{Estimate of the Basic Reproduction Number}

Using the next-generation matrix (NGM) approach described in [40] (see also [17]), we compute the basic reproduction number $\mathcal{R}_{0}$, which is define in our case as the number of secondary infections that an infectious pod could produce when introduced in a population of healthy pods of any maturity stage.

Following [40], we rewrite system (2) as follow

$$
\frac{d X}{d t}=f(X)=\mathcal{F}(X)-\mathcal{V}(X)
$$

where $X=\left(S_{1}, S_{2}, I, S_{p, i}, S_{p, e}\right)^{T}, \mathcal{F}$ is the incidence rate of new infections, and $\mathcal{V}$ is the transfer rate of individuals into, and out of, each sub-population. Let $\mathbf{X}_{s}=\left\{X \geq 0 / X_{i}=0, i=3, \ldots, 5\right\}$ be the set of all disease free states.

As it is very clearly explained in $[40,39,15]$, the previous decomposition is not unique, such that different choices for $\mathcal{F}$ lead to different values for the basic reproduction number. What is important is the choice that is epidemiologically relevant. According to field experts, and as explained in section 2, we distinguish $S_{p, i}$, spores that responsible for primary infections, from $S_{p, e}$, spores that are responsible to secondary infections. In other words $S_{p, e}$ acts as a reservoir, while $S_{p, i}$ is considered as an extension of the infectious pod compartment. Thus, according to these explanations, this leads to the following choices for $\mathcal{F}$ and $\mathcal{V}$ :

$$
\mathcal{F}(X)=\left(\begin{array}{c}
0 \\
0 \\
{\left[\frac{\beta_{1}^{1} S_{p, e}}{K_{1}+S_{p, e}}+\frac{\beta_{2}^{1} S_{p, i}}{K_{2}+S_{p, i}}\right] S_{1}+\left[\frac{\beta_{1}^{2} S_{p, e}}{K_{1}+S_{p, e}}+\frac{\beta_{2}^{2} S_{p, i}}{K_{2}+S_{p, i}}\right] S_{2}} \\
0 \\
r_{1} \sigma I
\end{array}\right),
$$

and $\mathcal{V}_{i}(X)_{=} \mathcal{V}_{i}^{-}(X)-\mathcal{V}_{+}(X)$, where

$$
\mathcal{V}_{i}^{-}(X)=\left(\begin{array}{c}
{\left[\frac{\beta_{1}^{1} S_{p, e}}{K_{1}+S_{p, e}}+\frac{\beta_{2}^{1} S_{p, i}}{K_{2}+S_{p, i}}+\left(\mu_{1}+\omega+\theta_{1}\right)\right] S_{1}} \\
{\left[\frac{\beta_{1}^{2} S_{p, e}}{K_{1}+S_{p, e}}+\frac{\beta_{2}^{2} S_{p, i}}{K_{2}+S_{p, i}}+\left(\mu_{2}+\theta_{2}\right)\right] S_{2}} \\
\left(\gamma_{I}+\mu_{I}\right) I \\
d_{2} S_{p, i} \\
d_{1} S_{p, e}
\end{array}\right) \quad \text { and } \mathcal{V}_{i}^{+}(X)=\left(\begin{array}{c}
0 \\
\theta_{1} S_{1} \\
0 \\
r_{2} \sigma I \\
0
\end{array}\right) \text {, }
$$

such that we verify the following assumptions given, for instance, in [40], that is

(A1) if $X \geq 0$, then $\mathcal{F}_{i}, \mathcal{V}_{i}^{+}$and $\mathcal{V}_{i}^{+} \geq 0$ for $i=1, \ldots, 5$.

(A2) if $X_{i}=0$, then $\mathcal{V}_{i}^{-}=0$.

(A3) $\mathcal{F}_{i}=0$ for $i=1, . ., 2$.

(A4) if $X \in \mathbf{X}_{s}$ then $\mathcal{F}_{i}=0$ and $\mathcal{V}_{i}^{+}=0$ for $i=3, . ., 5$ 
(A5) If $\mathcal{F}(X)$ is set to zero, then all eigenvalues of $D f_{D F E}$ have negative real parts. Indeed, we have

$$
D f_{D F E}=\left(\begin{array}{ccccc}
-\left(\mu_{1}+\omega+\theta_{1}\right) & 0 & 0 & \frac{\beta_{2}^{1} S_{1}^{D F E}}{K_{2}} & \frac{\beta_{1}^{1} S_{1}^{D F E}}{K_{1}} \\
\theta_{1} & -\left(\mu_{2}+\theta_{2}\right) & 0 & \frac{\beta_{2}^{2} S_{2}^{D F E}}{K_{2}} & \frac{\beta_{1}^{2} S_{2}^{D F E}}{K_{1}} \\
0 & 0 & -\left(\gamma_{I}+\mu_{I}\right) & 0 & 0 \\
0 & 0 & r_{2} \sigma & -d_{2} & 0 \\
0 & 0 & 0 & 0 & -d_{1}
\end{array}\right)
$$

A rapid calculation shows that all eigenvalues are real and negative (the terms in the diagonal), ensuring that $D F E$ is Locally Asymptotically Stable.

Then, considering the disease-free equilibrium, $D F E$, and taking into account that $f$ verifies assumptions (A1)-(A5), we can define the following $3 \times 3$ matrices

$$
F_{i, j}=\left.\frac{\partial \mathcal{F}_{i+2}}{\partial X_{j+2}}\right|_{D F E}, \quad 1 \leq i, j \leq 3
$$

and

$$
V_{i, j}=\left.\frac{\partial \mathcal{V}_{i+2}}{\partial X_{j+2}}\right|_{D F E}, \quad 1 \leq i, j \leq 3
$$

such that we obtain

$$
F=\left(\begin{array}{ccc}
0 & \frac{\beta_{2}^{1} S_{1}^{d f e}+\beta_{2}^{2} S_{2}^{d f e}}{K_{2}} & \frac{\beta_{1}^{1} S_{1}^{d f e}+\beta_{1}^{2} S_{2}^{d f e}}{K_{1}} \\
0 & 0 & 0 \\
r_{1} \sigma & 0 & 0
\end{array}\right), \quad V=\left(\begin{array}{ccc}
\gamma_{I}+\mu_{I} & 0 & 0 \\
-r_{2} \sigma & d_{2} & 0 \\
0 & 0 & d_{1}
\end{array}\right)
$$

$F$ is entrywise non-negative and $V$ is obviously a non-singular M-matrix, so that $V^{-1} \geq 0$. Thus, if we consider that $\Psi(0)$ is the number of initially infected individuals, then $F V^{-1} \Psi(0)(\geq 0)$ gives the expected number of new infections. $F V^{-1}$ is called the Next Generation Matrix. According to the previous computations, we derive

$$
F V^{-1}=\left(\begin{array}{ccc}
\frac{\left(\beta_{2}^{1} S_{1}^{d f e}+\beta_{2}^{2} S_{2}^{d f e}\right) r_{2} \sigma}{K_{2} d_{2}\left(\gamma_{i}+\mu_{i}\right)} & \frac{\beta_{2}^{1} S_{1}^{d f e}+\beta_{2}^{2} S_{2}^{d f e}}{K_{2} d_{2}} & \frac{\beta_{1}^{1} S_{1}^{d f e}+\beta_{1}^{2} S_{2}^{d f e}}{K_{1} d_{1}} \\
\frac{r_{1} \sigma}{\gamma_{i}+\mu_{i}} & 0 & 0 \\
0 & 0 & 0
\end{array}\right)
$$

According to [40], the basic reproduction number, $\mathcal{R}_{0}$, is defined as the spectral radius of $F V^{-1}$, i.e. $\mathcal{R}_{0}=\rho\left(F V^{-1}\right)$. A straightforward computation shows that the characteristic polynomial of $F V^{-1}$ is

$$
P(\lambda)=\lambda\left[\mathcal{R}_{0, d} \lambda+\mathcal{R}_{0, i}-\lambda^{2}\right]
$$

where

$$
\mathcal{R}_{0, d}=\frac{r_{2} \sigma\left(\beta_{2}^{1} S_{1}^{d f e}+\beta_{2}^{2} S_{2}^{d f e}\right)}{K_{2} d_{2}\left(\gamma_{i}+\mu_{i}\right)} \quad \text { and } \quad \mathcal{R}_{0, i}=\frac{r_{1} \sigma\left(\beta_{1}^{1} S_{1}^{d f e}+\beta_{1}^{2} S_{2}^{d f e}\right)}{K_{1} d_{1}\left(\gamma_{i}+\mu_{i}\right)} .
$$

The eigenvalues of $F V^{-1}$ are the roots of $P$, and the spectral radius the largest ones, which leads to

$$
\mathcal{R}_{0}=\frac{1}{2}\left(\mathcal{R}_{0, d}+\sqrt{\left(\mathcal{R}_{0, d}\right)^{2}+4 \mathcal{R}_{0, i}}\right)
$$


Remark 1. Note that $\mathcal{R}_{0}$ depends on $\mathcal{R}_{0, d}$ and $\mathcal{R}_{0, i}$, that can be seen respectively as the basic reproduction number related each transmission route: the direct route (pods to pods) and indirect route (environment to pods).

Following [40], we have

Proposition 1. DFE is locally asymptotically stable when $\mathcal{R}_{0}<1$ and unstable when $\mathcal{R}_{0}>1$.

It is important to recall that local asymptotic stability of the disease-free equilibrium does not guarantee complete elimination of the disease. Only global asymptotic stability ensures that the disease either dies out or persists.

Remark 2. Last but not least, if $S_{p, e}$ is not seen as a reservoir compartment, but simply as a transition compartment, then the Next Generation Matrix becomes

$$
F V^{-1}=\left(\begin{array}{ccc}
\mathcal{R}_{0, d}+\mathcal{R}_{0, i} & \frac{\beta_{2}^{1} S_{1}^{d f e}+\beta_{2}^{2} S_{2}^{d f e}}{K_{2} d_{2}} & \frac{\beta_{1}^{1} S_{1}^{d f e}+\beta_{1}^{2} S_{2}^{d f e}}{K_{1} d_{1}} \\
0 & 0 & 0 \\
0 & 0 & 0
\end{array}\right),
$$

such that the two routes of transmission enter $\mathcal{R}_{0}$ in an additive way, i.e. $\mathcal{R}_{0, g}=\mathcal{R}_{0, d}+\mathcal{R}_{0, i}$. However, according to [7], or [39], or [40], both thresholds are mathematically equivalent, i.e. they are simultaneously greater, equal or less than one. See also [15], where the authors discussed the several forms of the basic reproduction number for continuous and discrete epidemiological (population) systems.

\subsubsection{Global Stability of the DFE}

To show the global stability of the DFE, we used a theorem proved by Kamgang and Sallet [25], and recalled, for convenience, in Appendix A. (see theorem 2, page 27)

Using the the DFE, we rewrite (2) in the following manner

$$
\left\{\begin{array}{l}
\dot{x}_{S}=A_{1}(x)\left(x_{S}-x_{D F E, S}\right)+A_{1,2}(x) x_{I}, \\
\dot{x}_{I}=A_{2}(x) x_{I}
\end{array}\right.
$$

where $x_{s}$ is the vector representing the state of different compartments of non transmitting individuals and the vector $x_{I}$ represents the state of compartments of different transmitting individuals. Here, we have $x_{s}=\left(S_{1}, S_{2}\right)^{T}, x_{I}=\left(I, S_{p, i}, S_{p, e}\right)^{T}$ and $x_{D F E, S}=\left(\frac{\Lambda}{\theta_{1}+\mu_{1}+w}, \frac{\theta_{1} \Lambda}{\mu_{2}\left(\theta_{1}+\mu_{1}+w\right)}\right)^{T}$. Indeed from $(S)$, we obtain the following matrices for $A_{1}(x), A_{1,2}(x)$ and $A_{2}(x)$

and

$$
\begin{gathered}
A_{1}(x)=\left(\begin{array}{cc}
-\left(\theta_{1}+\mu_{1}+w\right) & 0 \\
\theta_{1} & -\mu_{2}-\theta_{2}
\end{array}\right) \\
A_{1,2}(x)=\left(\begin{array}{ccc}
0 & \frac{-\beta_{2}^{1} S_{1}}{K_{2}} & \frac{-\beta_{1}^{1} S_{1}}{K_{1}} \\
0 & \frac{-\beta_{2}^{2} S_{2}}{K_{2}} & \frac{-\beta_{1}^{2} S_{2}}{K_{1}}
\end{array}\right)
\end{gathered}
$$

$$
A_{2}(x)=\left(\begin{array}{ccc}
-\left(\gamma_{I}+\mu_{I}\right) & \frac{\beta_{2}^{1} S_{1}+\beta_{2}^{2} S_{2}}{K_{2}} & \frac{\beta_{1}^{1} S_{1}+\beta_{1}^{2} S_{2}}{K_{1}} \\
r_{2} \sigma & -d_{2} & 0 \\
r_{1} \sigma & 0 & -d_{1}
\end{array}\right) .
$$

Following [25], we show the global asymptotically stability of the $D F E$ and we derive the following theorem 
Theorem 1. The disease free equilibrium of system (2) is globally asymptotically stable if $\mathcal{R}_{0} \leq 1$.

Proof. see Appendix B.

Remark 3. The previous result also holds if $\mathcal{R}_{0}$ is replaced by $\mathcal{R}_{0, g}$. We will see that $\mathcal{R}_{0, g}$ plays a role in the existence of at least one endemic equilibrium.

\subsubsection{About Endemic equilibria}

To derive the endemic equilibria, we solve system (5), page 8 , which leads to

$$
\begin{cases}S_{1}^{*} & =\frac{\Lambda}{\left[\frac{\beta_{1}^{1} r_{1} \sigma I^{*}}{K_{1} d_{1}+r_{1} \sigma I^{*}}+\frac{\beta_{2}^{1} r_{2} \sigma I^{*}}{K_{2} d_{2}+r_{2} \sigma I^{*}}\right]+\theta_{1}+\mu_{1}+w} \\ & =\frac{\Lambda \theta_{1}}{\left(\left[\frac{\beta_{1}^{1} r_{1} \sigma I^{*}}{K_{1} d_{1}+r_{1} \sigma I^{*}}+\frac{\beta_{2}^{1} r_{2} \sigma I^{*}}{K_{2} d_{2}+r_{2} \sigma I^{*}}\right]+\theta_{1}+\mu_{1}+w\right)\left(\left[\frac{\beta_{1}^{2} r_{1} \sigma I^{*}}{K_{1} d_{1}+r_{1} \sigma I^{*}}+\frac{\beta_{2}^{2} r_{2} \sigma I^{*}}{K_{2} d_{2}+r_{2} \sigma I^{*}}\right]+\mu_{2}+\theta_{2}\right)} \\ \left(\gamma_{I}+\mu_{I}\right) I^{*} & =\left[\frac{\beta_{1}^{1} r_{1} \sigma I^{*}}{K_{1} d_{1}+r_{1} \sigma I^{*}}+\frac{\beta_{2}^{1} r_{2} \sigma I^{*}}{K_{2} d_{2}+r_{2} \sigma I^{*}}\right] S_{1}^{*}+\left[\frac{\beta_{1}^{2} r_{1} \sigma I^{*}}{K_{1} d_{1}+r_{1} \sigma I^{*}}+\frac{\beta_{2}^{2} r_{2} \sigma I^{*}}{K_{2} d_{2}+r_{2} \sigma I^{*}}\right] S_{2}^{*} \\ S_{p, i}^{*} & =\frac{r_{2} \sigma}{d_{2}} I^{*} \\ S_{p, e}^{*} & =\frac{r_{1} \sigma}{d_{1}} I^{*} .\end{cases}
$$

Then, replacing $(9)_{1}$ and $(9)_{2}$ in the right-hand side of $(9)_{3}$, leads to look after the positive roots of the following polynomial

$$
P(I)=a_{4} I^{4}+a_{3} I^{3}+a_{2} I^{2}+a_{1} I+a_{0},
$$

where the terms $\left(a_{i}\right)_{0 \leq i \leq 4}$ are given in Appendix C, page 30. In particular, we show that

$$
a_{0}=\left(\gamma_{I}+\mu_{I}\right) B_{0}^{2}\left[1-\mathcal{R}_{0, g}\right],
$$

such that we are able to deduce

Lemma 1. The sign of $a_{0}$ is related to $\mathcal{R}_{0, g}$ as follows:

1. $a_{0}<0$ is equivalent to $\mathcal{R}_{0, g}>1$

2. $a_{0}>0$ is equivalent to $\mathcal{R}_{0, g}<1$.

Then, according to Descarte's rule of sign (see Appendix C, page 30), we deduce

Proposition 2. When $\mathcal{R}_{0, g}>1$ system (2) admits one or three endemic equilibria.

Remark 4. The study of the stability/instability of the endemic equilibrium is not straightforward. Therefore, we will only check numerically the stability property in the forthcoming simulations.

Since $\mathcal{R}_{0}$ and $\mathcal{R}_{0, g}$ are equivalent, proposition 2 leads to

Proposition 3. When $\mathcal{R}_{0}>1$ system (2) admits one or three endemic equilibria. 


\subsection{The Phytophthora model with periodic recruitment rate}

When $\Lambda$ is periodic (or if we consider that all parameters are periodic), of period $T$, the previous estimates on the basic reproduction numbers are no more available. We still have existence of a nonnegative bounded solution. It is also straightforward to show that system (2), with periodic recruitment rate, admits an unique $T$-periodic disease free solution, $P D F E=\left(S_{1, p e r}(t), S_{2, p e r}(t), 0,0,0\right)$, where

$$
S_{1, p e r}(t)=\left(\frac{1}{e^{\left(\theta_{1}+\mu_{1}+w\right) T}-1} \int_{0}^{T} \Lambda(\tau) e^{\left(\theta_{1}+\mu_{1}+w\right) \tau} d \tau+\int_{0}^{t} \Lambda(\tau) e^{\left(\theta_{1}+\mu_{1}+w\right) \tau} d \tau\right) e^{-\left(\theta_{1}+\mu_{1}+w\right) t}
$$

and

$$
S_{2, p e r}(t)=\left(\frac{1}{e^{\left(\mu_{2}+\theta_{2}\right) T}-1} \int_{0}^{T} S_{1, p e r}(\tau) e^{\left(\mu_{2}+\theta_{2}\right) \tau} d \tau+\int_{0}^{t} S_{1, p e r}(\tau) e^{\left(\mu_{2}+\theta_{2}\right) \tau} d \tau\right) e^{-\mu_{1} t}
$$

Following [42], we are able to estimate a basic reproduction number, $\mathcal{R}_{0, p e r}$ related to $P D F E$. In Appendix A, we recall briefly the main assumptions, (A1)-(A6), to verify to be able to apply Proposition 4, page 28 (see also [42]). Note that most of these assumptions are similar to those given in [40] to estimate the standard basic reproduction number for constant parameters. However, in the periodic case, they are two additional ones, (A6) and (A7) that we can easily verify here. Indeed, since $-V$ is a lower triangular cooperative matrix with constant values, the monodromy matrix of system $\frac{d y}{d t}=-V y$ is given by

$$
\Phi_{-V}(t)=\left(\begin{array}{ccc}
e^{-\left(\gamma_{I}+\mu_{I}\right) t} & 0 & 0 \\
* & e^{-d_{2} \tau} & 0 \\
0 & * & e^{-d_{1} t}
\end{array}\right)
$$

Thus we deduce that $\rho\left(\Phi_{-V}(T)\right)<1$. Assumption (A7) is verified. Let us verify assumption (A6). We compute

$$
M(t)=\left(\begin{array}{cc}
-\left(\theta_{1}+\mu_{1}+w\right) & 0 \\
\theta_{1} & -\mu_{2}-\theta_{2}
\end{array}\right) .
$$

Again, $M(t)$ being a lower triangular matrix with constant parameters, we deduce that the monodromy matrix $\Phi_{M}(t)$ is defined as follows

$$
\Phi_{M}(t)=\left(\begin{array}{cc}
e^{-\left(\theta_{1}+\mu_{1}+w\right) t} & 0 \\
* & e^{-\left(\mu_{2}+\theta_{2}\right) t}
\end{array}\right) .
$$

such that $\rho\left(\Phi_{M}(T)\right)<1$. Assumptions (A6) and (A7) being verified, PDFE is asymptotically stable. Following [42] (see appendix A) we consider the following linear $\tau$-periodic system

$$
\frac{d w}{d t}=\left(-V+\frac{F(t)}{\lambda}\right) w
$$

with parameter $\lambda \in(0, \infty)$, and

$$
F(t)=\left(\begin{array}{ccc}
0 & \frac{\beta_{2}^{1} S_{1, p e r}(t)+\beta_{2}^{2} S_{2, p e r}(t)}{K_{2}} & \frac{\beta_{1}^{1} S_{1, p e r}(t)+\beta_{1}^{2} S_{2, p e r}(t)}{K_{1}} \\
0 & 0 & 0 \\
r_{1} \sigma & 0 & 0
\end{array}\right)
$$


and

$$
V=\left(\begin{array}{ccc}
\gamma_{I}+\mu_{I} & 0 & 0 \\
-r_{2} \sigma & d_{2} & 0 \\
0 & 0 & d_{1}
\end{array}\right)
$$

For each $\lambda>0$, we are able to estimate the monodromy matrix $W(\tau, \lambda)$ and thus its spectral radius, $\rho(W(\tau, \lambda))$. According to Theorem 2.1 [42] (see also Appendix A), we are looking for $\lambda^{*}>0$ such that $\rho\left(W\left(\tau, \lambda^{*}\right)\right)=1$, and thus deduce $\mathcal{R}_{0, p e r}$, the local periodic basic reproduction number. Thanks to remark 2 , page 11 , we can also define similarly $\mathcal{R}_{0, g, p e r}$. Numerical estimates of $\mathcal{R}_{0, p e r}$ and $\mathcal{R}_{0, g, p e r}$ are obtained by solving (10), using an iterative algorithm, as explained in the end of Appendix A.

\subsection{Global Sensitivity analysis of the Phytophthora model}

A full sensitivity analysis is performed on the model outputs by using two well-known methods: LHS-PRCC (Latin hypercube sampling-partial rank correlation coefficient) and eFAST (Extended Fourier amplitude sensitivity test) [28]. These are complementary methods: PRCC provides mainly information about how the outputs are impacted if we increase (or decrease) the inputs of a specific parameter while eFAST indicates which parameter uncertainty has the greatest impact on the output variability (see[28] for further explanations). We consider the ranges of values given in Table 4, page 36. For some parameters, we have a relative good idea about the range of values, for others, like the spores parameters, this is more vague, but we choose value that, according to filed experts, are the most reasonable. In any case, and whatever the methods used to make the full sensitivity analysis, the results are discussed and valid only under the chosen intervals.

First, in Fig. 4, page 15, we show a LHS-PRCC analysis on both basic reproduction numbers, $\mathcal{R}_{0}$ and $\mathcal{R}_{0, g}$. The parameters impact negatively or positively, as expected. However, it is interesting to observe that, $\mathcal{R}_{0}$, is more impacted specifically by parameters related to the indirect transmission, that is $K_{1}, r_{1}$ and $d_{1}$.

Sensitivity analysis results are given in Fig. 5, page 16, and Fig. 6, page 17. While for the variables $S_{1}$ and $S_{2}$, both methods provide the same analysis, that is $\Lambda, \omega, \mu_{i}$, and $\theta_{i}$ are very sensitive parameters, the results are more contrasted in the compartments related to the dynamics of the disease. However, from both methods it is clear that for $I, S p_{i}$ and $S p_{e}, d_{1}$ and $d_{2}$ are very sensitive parameters, $\gamma_{i}$, the roguing parameter, $\Lambda$, and $\sigma$ are also sensitive parameters. Surprisingly $\beta_{i j}$ are not so sensitive as expected. However, from the eFAST, the $K_{i}$ parameters seem to be very sensitive. It is interesting to emphasize that given the sensitivity of the death rate parameters $d_{i}$, it makes sense to use a control method like fungicide. This is in good agreement with ongoing field practice.

Altogether, this brief sensitivity study shows that not only the parameters related to the dynamics of the pods are important, but also the parameters related to the dynamics of the spores. While, the pod population dynamic is relatively well known, thanks to previous studies, this seems not the case for the spores, where clearly the mean lifespan, $d_{i}$, as well as the spores production by pods, $r_{i}$, is not very well known.

\section{Numerical simulations and discussions}

We now provide and discuss some numerical simulations to illustrate the previous results and further. The numerical approximations are obtained using Matlab 2016. Since for several parameters 
LHS-PRCC Stability Analysis- Phytophthora model - $\mathrm{R}_{0}$

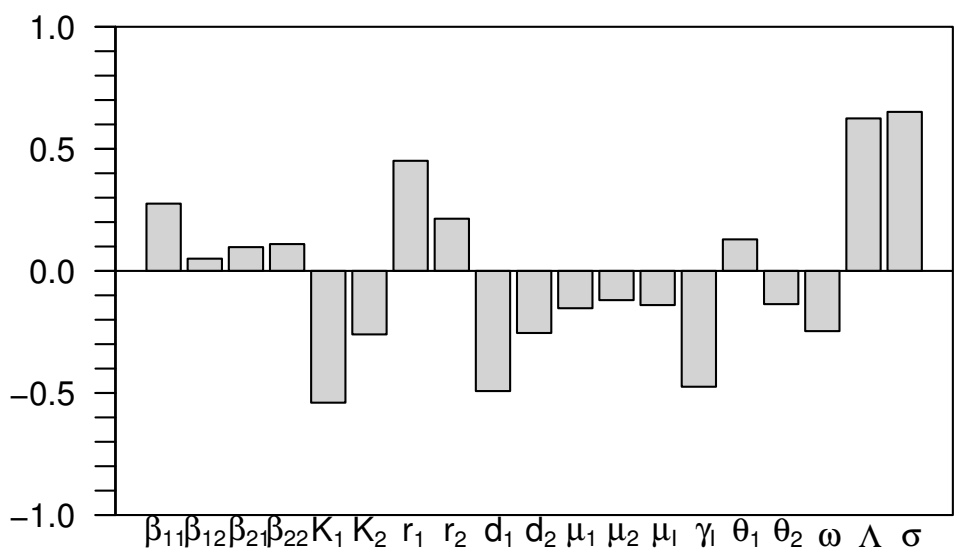

LHS-PRCC Stability Analysis- Phytophthora model - $\mathrm{R}_{0}$ (global)

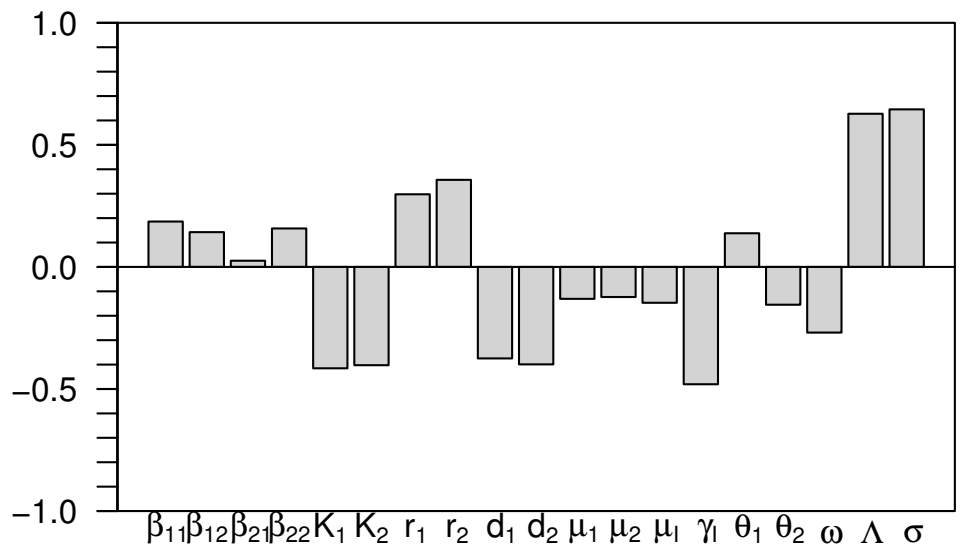

Figure 4: LHS-PRCC sensitivity analysis of $\mathcal{R}_{0}$ and $\mathcal{R}_{0, g}$.

little to no data are available (yet) in the literature, estimations of these parameters for use in the simulations were based on expert knowledge of the pathosystem. The range of values of model's parameters are mentioned in table 1 , page 33 , in section 1 .

According to these parameters, when $\gamma_{I}=0$, we have $\mathcal{R}_{0}>1$ (or $\mathcal{R}_{0, g}>1$ ), such that system (2) admits a unique endemic equilibrium that is Locally Asymptotically Stable, at least numerically. 
eFast Stability Analysis- Phytophthora model - $\mathrm{S}_{1}$

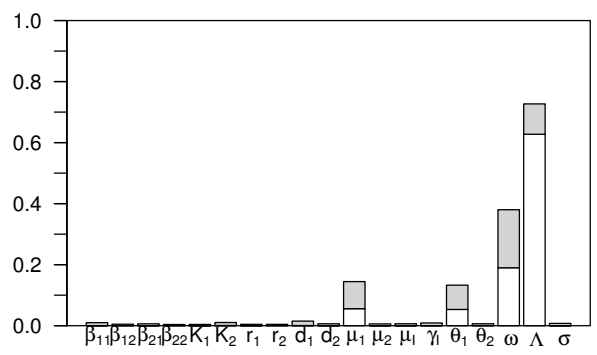

eFast Stability Analysis- Phytophthora model - I

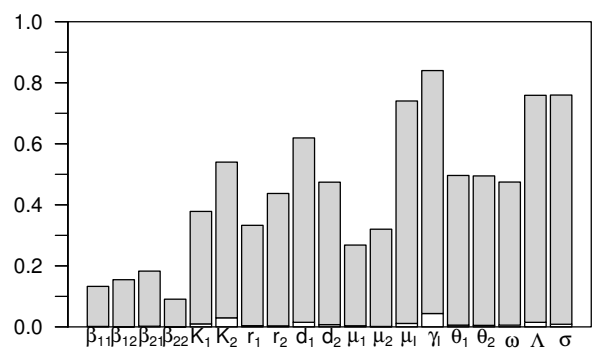

eFast Stability Analysis- Phytophthora model $-\mathrm{S}_{2}$

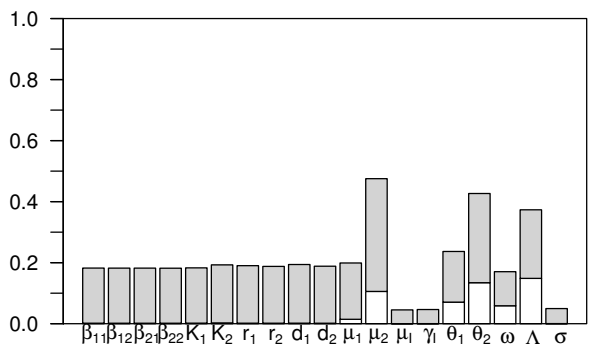

eFast Stability Analysis- Phytophthora model $-\mathrm{Sp}_{\mathrm{e}}$

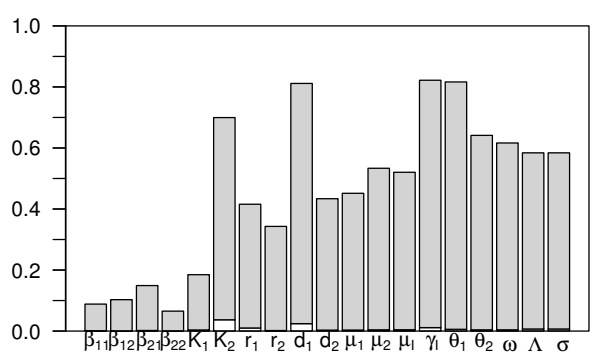

Figure 5: e-FAST sensitivity analysis of Model's outputs. White bar: first-order effects; Sum of white and grey bars: total effect.

\subsection{Estimation of the cherelle recruitment rate, $\Lambda$}

In the context of Cameroon, it is not realistic to consider a constant recruitment rate for cherelles. In contrary, cocoa pods are strongly connected to rainy periods: a long and a short rainy season. Thus, using data from the literature [10], we estimate $\Lambda$ as a $T$-periodic function (see Fig. 7, page 18 ), where $T=365$ days. 
LHS-PRCC Stability Analysis- Phytophthora model - $\mathrm{S}_{1}$

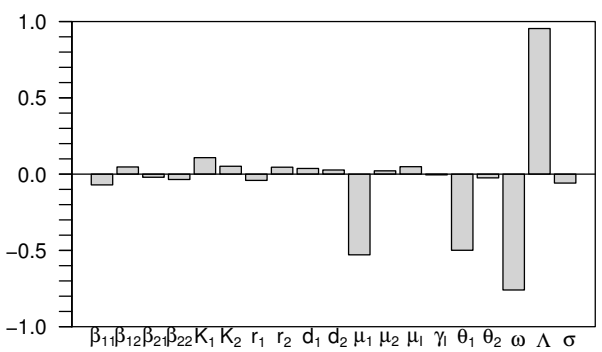

LHS-PRCC Stability Analysis- Phytophthora model - I

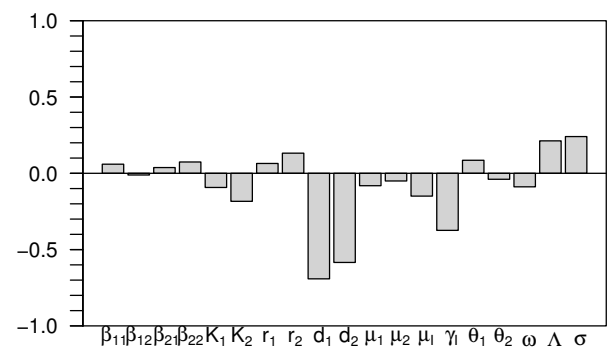

LHS-PRCC Stability Analysis- Phytophthora model $-\mathrm{S}_{2}$

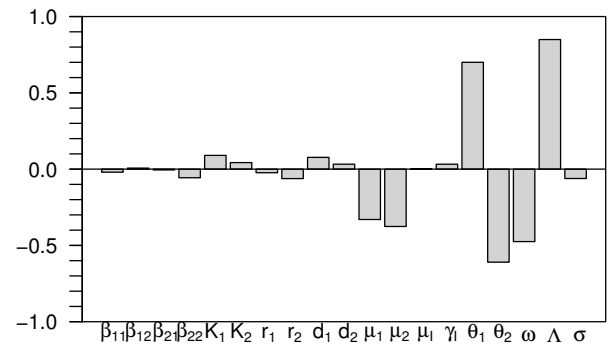

LHS-PRCC Stability Analysis- Phytophthora model - $\mathrm{Sp}_{\mathrm{i}}$

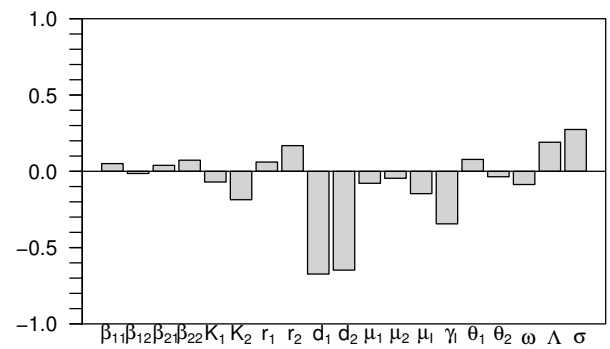

Figure 6: LHS-PRCC sensitivity analysis of Model's outputs

\subsection{Impact of phytosanitary pod removal on the disease dynamic}

Using parameters values given in table 1, page 33, we highlight the impact of phytosanitary pod removal on the disease dynamic of the system in two specific situations: when $\Lambda$ is constant, and when $\Lambda$ is a time periodic function, defined in Fig.7, page 18. In the next simulations, we highlight the dynamics without sanitary harvest and with sanitary harvest.

\subsubsection{Simulations with a constant recruitment rate $\Lambda$}

As value for $\Lambda$, we consider the mean value of $\Lambda_{\text {per }}$ over one period, i.e. one year, i.e $\Lambda=$ $\frac{1}{365} \int_{0}^{365} \lambda_{\text {per }}(t) d t \approx 12$. In Figs. 8 and 9 , page 18 , we consider two initial conditions: when 


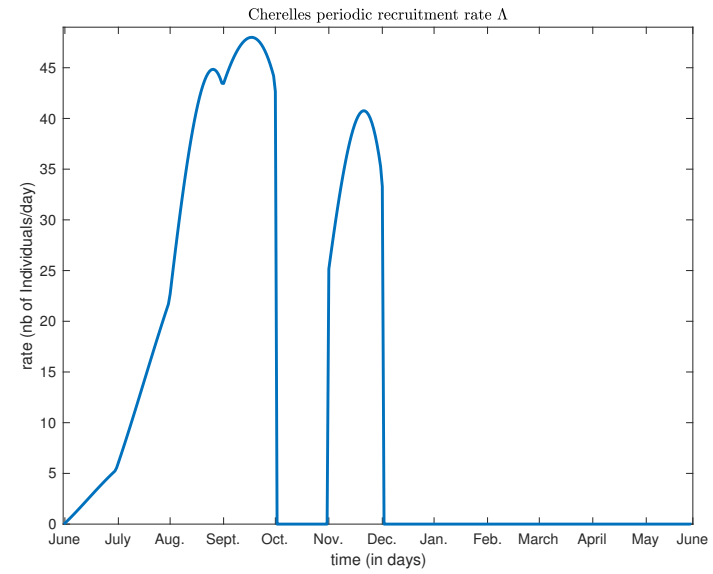

Figure 7: Cherelle recruitment rate function in the year

one pod is infectious, and when spores are present in the environment. Both simulations illustrate clearly the impact of the phytosanitary pod removal on disease dynamics. Indeed, the application of sanitary harvest $(t>750)$, i.e. $\gamma=0.25$, drives $\mathcal{R}_{0}$ from a value greater than one to a value lower than 1, such that the DFE becomes globally asymptotically stable, i.e. the infection dies out. It is also interesting to notice that according to the initial conditions, the system, while converging to the same constant positive equilibrium, may have a different transient dynamic.
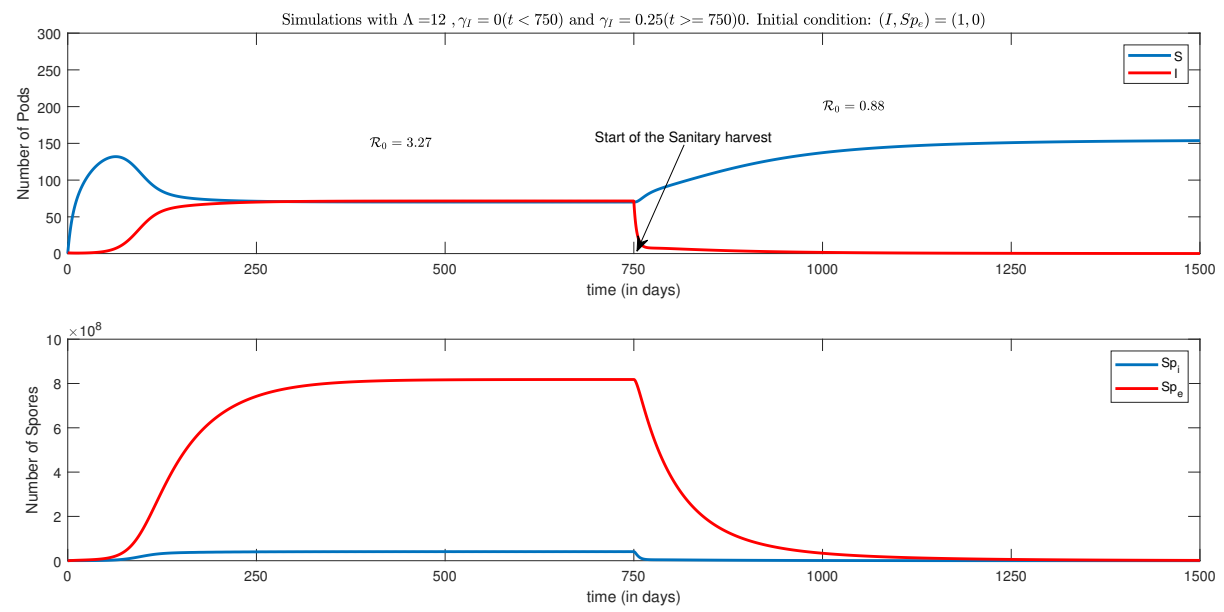

Figure 8: Time evolution of Susceptible pods, Infected pods, and Spores, when the initial infection starts with an infective pod. $\Lambda=12, \gamma_{I}=0(t<750)$ and $\gamma_{I}=0.25(t>750)$.

\subsubsection{Simulations with a periodic recruitment rate $\Lambda(t)$}

In Figs.10 and 11, we show simulations with $\Lambda(t)$ periodic, as defined in Fig.7, page 18, and for different sanitary harvest rate, $\gamma_{I}=0,0.25$, and 0.3 . Using the same parameters values, but with a periodic cherelle recruitment rate, the phytosanitary pod removal, with $\gamma_{I}=0.25$, is clearly efficient in reducing the number of infected pods and spores. However, $\mathcal{R}_{0, p e r}$ is already greater than 1, and thus, contrary to the constant case, the disease does not stop (see Table 2, page 33). 

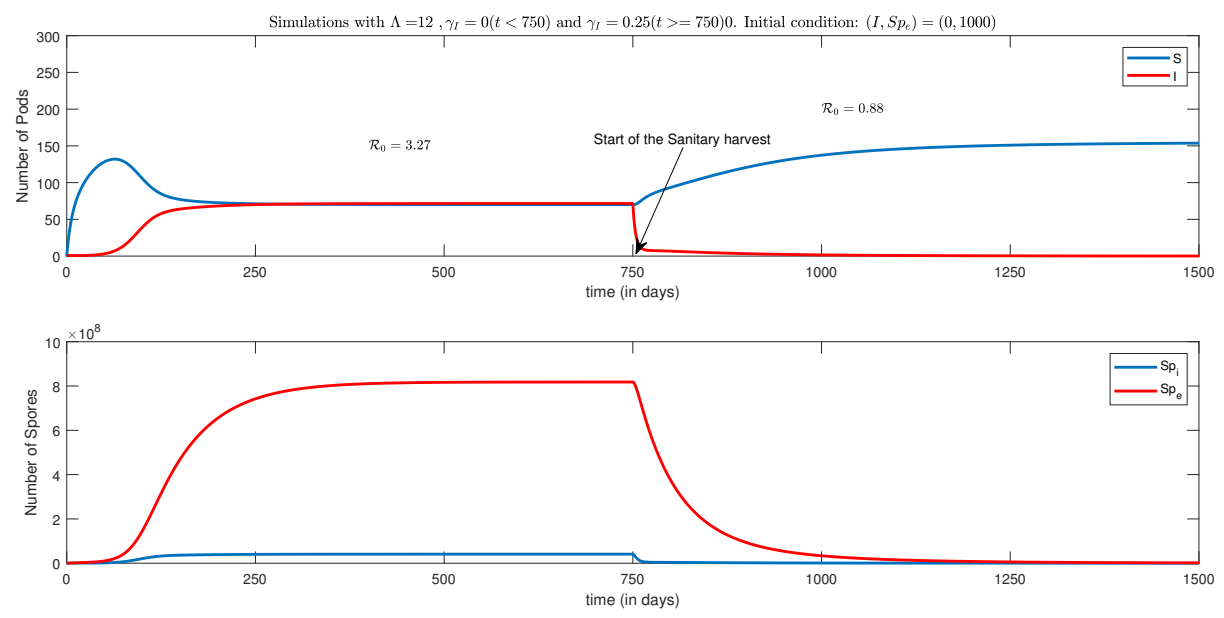

Figure 9: Time evolution of Susceptible pods, Infected pods, and Spores, when the initial infection starts with spores in the environment, $S_{p e}=1000 . \Lambda=12, \gamma_{I}=0(t<750)$ and $\gamma_{I}=0.25(t>750)$.
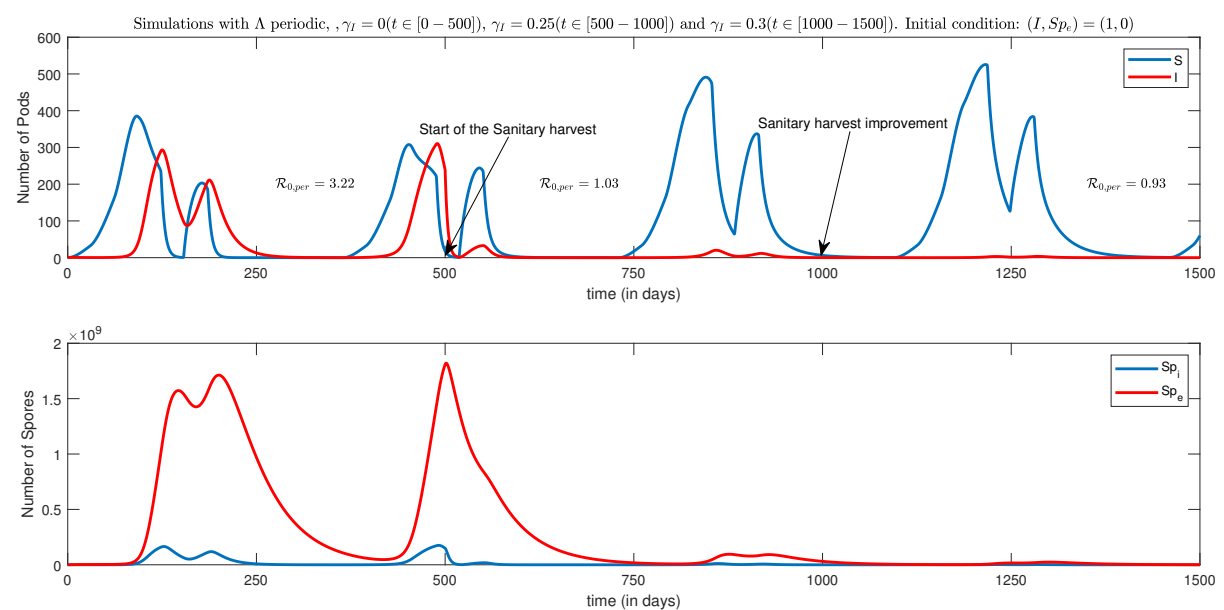

Figure 10: Periodic time evolution of Susceptible pods, Infected pods, and spores, when the initial infection starts with an infective pod and increasing sanitary harvest intensity. $\Lambda$ periodic, $\gamma_{I}=$ $0(t \in[0-500]), \gamma_{I}=0.25(t \in[500-1000])$ and $\gamma_{I}=0.3(t \in[1000-1500]$

This is related to the use of a periodic recruitment rate and shows clearly that considering $\lambda$ as a constant may give wrong results in terms of control strategies. Like in the constant case, there is a clear difference in the dynamic of the outbreaks when initially, either one pod is infected or spores are present in the environment. The disease spreads faster when a pod is initially infected. Finally, as expected, increasing the sanitary harvest, $\gamma_{I}=0.3$, decays $\mathcal{R}_{0, p e r}$ below 1 , such that the disease dies out. We summarize the values computed for the basic reproduction numbers in Table 2, page 33.

\subsubsection{Impact of periodic impulsive sanitary harvest on disease dynamics}

Here, instead of considering a continuous sanitary harvest, we consider that the sanitary harvest occurs instantaneously (impulsive events), with a periodicity, $\tau$, of 4,7 , and 14 days respectively. 

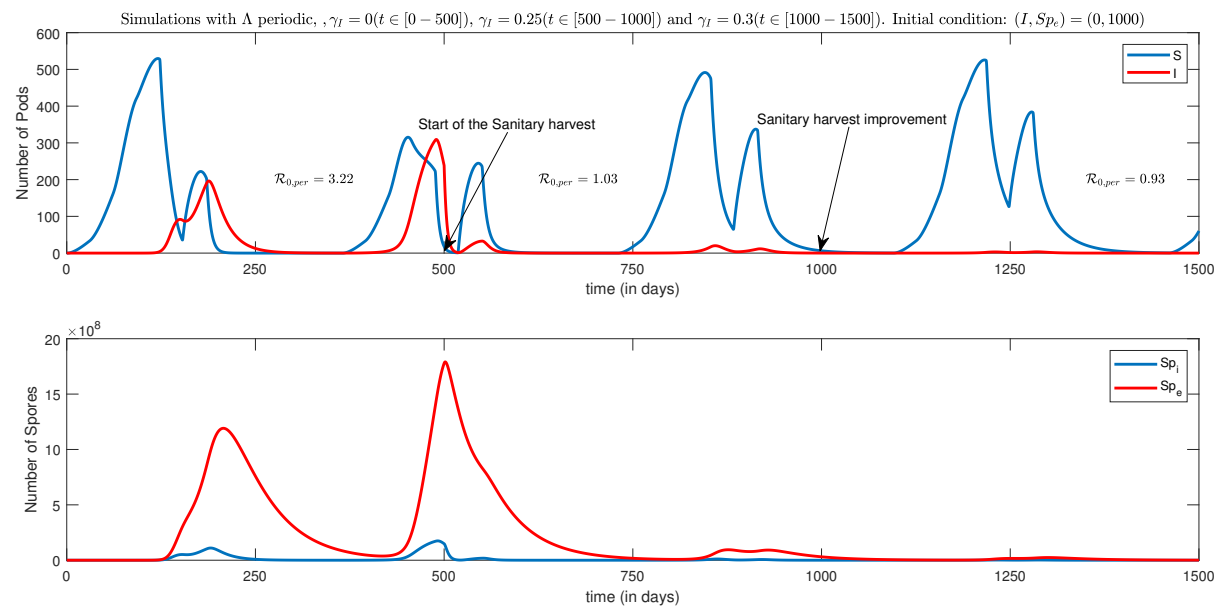

Figure 11: Periodic time evolution of Susceptible pods, Infected pods, and spores, when the initial infection starts with spores in the environment, $S_{p e}=1000$ and increasing sanitary harvest intensity. $\Lambda$ periodic, $\gamma_{I}=0(t \in[0-500]), \gamma_{I}=0.25(t \in[500-1000])$ and $\gamma_{I}=0.3(t \in[1000-1500]$

Compared to what happens in the field, this modeling is more realistic. In other words, we assume that a proportion, $\left.\Gamma_{I} \in\right] 0,1[$, of infected pods, $I$, is removed instantaneously every $\tau$ days. This leads to the following system

$$
\left\{\begin{aligned}
\frac{d S_{1}}{d t} & =\Lambda-\theta_{1} S_{1}-\left[\frac{\beta_{1}^{1} S_{p, e}}{K_{1}+S_{p, e}}+\frac{\beta_{2}^{1} S_{p, i}}{K_{2}+S_{p, i}}\right] S_{1}-\mu_{1} S_{1}-w S_{1} \\
\frac{d S_{2}}{d t} & =\theta_{1} S_{1}-\left[\frac{\beta_{1}^{2} S_{p, e}}{K_{1}+S_{p, e}}+\frac{\beta_{2}^{2} S_{p, i}}{K_{2}+S_{p, i}}\right] S_{2}-\mu_{2} S_{2} \\
\frac{d I}{d t} & =\left[\frac{\beta_{1}^{1} S_{p, e}}{K_{1}+S_{p, e}}+\frac{\beta_{2}^{1} S_{p, i}}{K_{2}+S_{p, i}}\right] S_{1}+\left[\frac{\beta_{1}^{2} S_{p, e}}{K_{1}+S_{p, e}}+\frac{\beta_{2}^{2} S_{p, i}}{K_{2}+S_{p, i}}\right] S_{2}-\mu_{I} I \\
\frac{d S_{p, i}}{d t} & =r_{2} \sigma I-d_{2} S_{p, i} \\
\frac{d S_{p, e}}{d t} & =r_{1} \sigma I-d_{1} S_{p, e}
\end{aligned}\right.
$$

with impulsive conditions

$$
\left\{\begin{aligned}
S_{1}\left(t_{s}+n \tau^{+}\right) & =S_{1}\left(t_{s}+n \tau\right), \\
S_{2}\left(t_{s}+n \tau^{+}\right) & =S_{2}\left(t_{s}+n \tau\right), \\
I\left(t_{s}+n \tau^{+}\right) & =\left(1-\Gamma_{I}\right) I\left(t_{s}+n \tau\right), \quad n \in \mathbb{N}^{*}, \\
S_{p, i}\left(t_{s}+n \tau^{+}\right) & =S_{p, i}\left(t_{s}+n \tau\right), \\
S_{p, e}\left(t_{s}+n \tau^{+}\right) & =S_{p, e}\left(t_{s}+n \tau\right),
\end{aligned}\right.
$$

where $t_{s}$ is the time when the (instantaneous) periodic sanitary harvest starts. According to the theory of impulsive differential equations [6], system (11)-(12) is well defined and admits a unique positive solution. However, at this stage, we don't want to enter too much in the theory. This is left for a future work.

In Fig. 12, page 21, Fig. 13, page 21, and Fig. 14, page 22, we obtain contrasting results. Indeed, considering a 14 or a 7 days sanitary harvest, even with $60 \%$ of removal, shows poor result compared to the period before the harvest (see Figs. 12 and 13). Only a 3-days harvest leads to significant results (see Fig. 14). Clearly periodicity and intensity of the harvest may impact the efficacy of this control method. From the field point of view, $60 \%$ of removal is quite large, but this may depend on the model's parameters. This requires further investigation. 

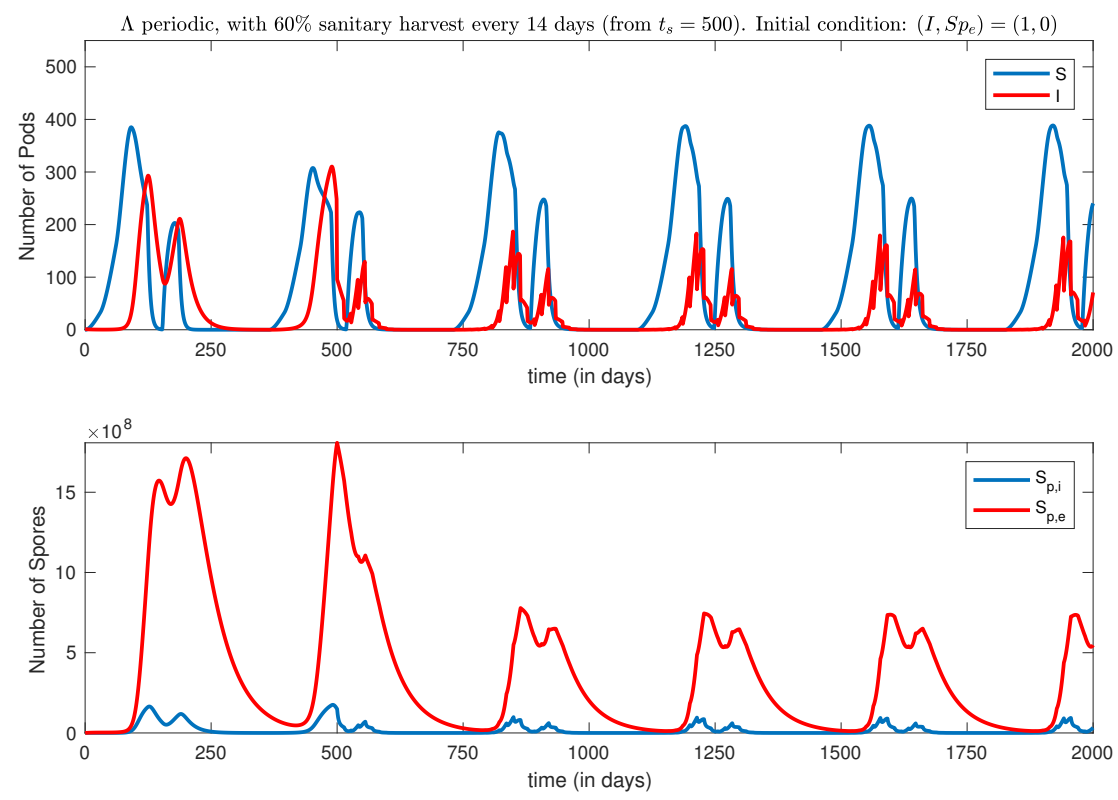

Figure 12: Periodic time evolution of Susceptible pods, Infected pods, and spores, when sanitary harvest starts at $t=500$, with a frequency of 14 days. $\Lambda$ periodic, and $\Gamma_{I}=0.6(t>500)$
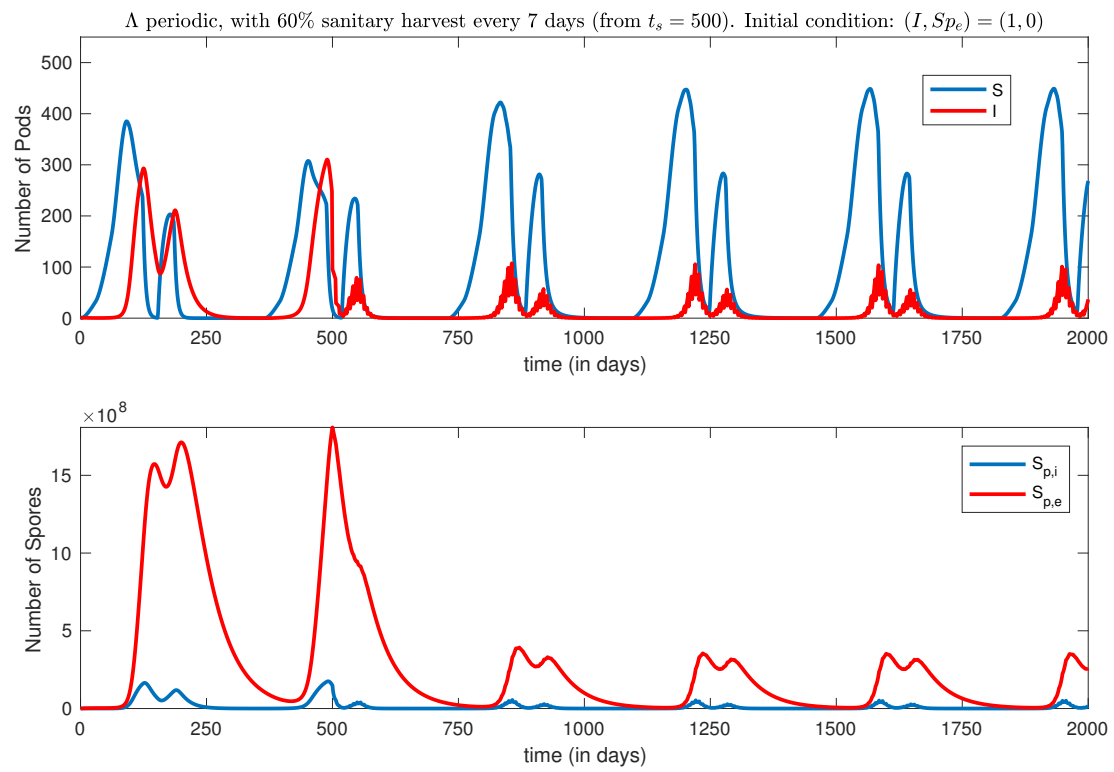

Figure 13: Periodic time evolution of Susceptible pods, Infected pods, and spores, when sanitary harvest starts at $t=500$, with a frequency of 7 days. $\Lambda$ periodic, and $\Gamma_{I}=0.6(t>500)$

\section{Discussion and Conclusion}

This paper considers an epidemiological model which incorporates a periodical birth rate function for cocoa pods and analyzes the temporal evolution of black pod disease in a plot. The impact 

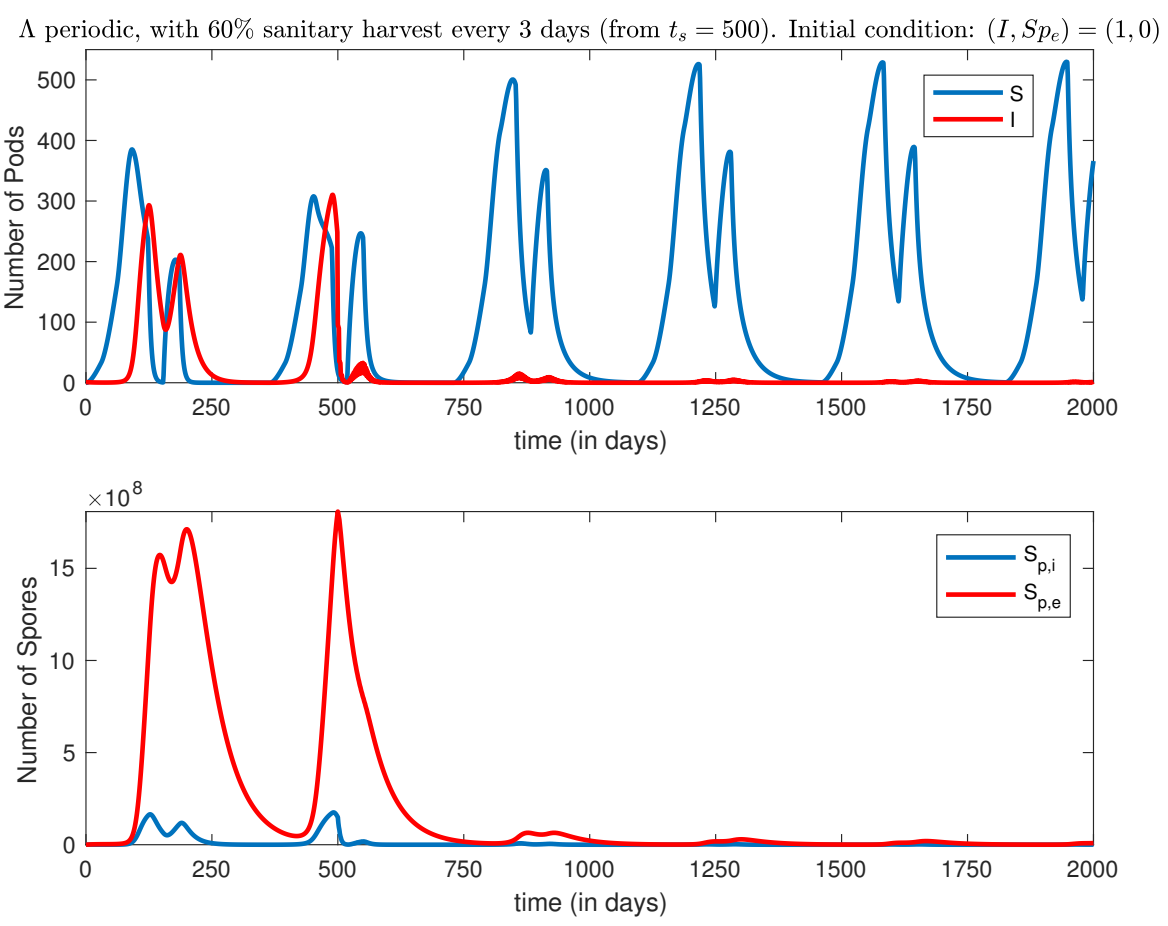

Figure 14: Periodic time evolution of Susceptible pods, Infected pods, and spores, when sanitary harvest starts at $t=500$, with a frequency of 3 days. $\Lambda$ periodic, and $\Gamma_{I}=0.6(t>500)$

of phytosanitary pod removal was assessed here and the results seem to be in general agreements with the literature. [30] shows that application of sanitary harvest could lead to reduced black pod incidence by $22 \%$ to $31 \%$, [33] found that weekly removal of infected pods by P. palmivora reduced the incidence of epidemics significantly in comparison with fortnightly removal (35 to $66 \%$ incidence reduction). Our results show that intense and regular phytosanitary pod removal (every four days) could reduce disease incidence even more than these percentages. In some cases number of infected pods in the plot could practically be reduced to nothing. It is important to mention that the good qualitative properties of the system can be partially explained by the fact that the model considers an isolated cocoa plot. Thus, we are neglecting external factors such as external inoculum and neighboring plots which can provide external sources of infections. Furthermore, the model does not take into account the impact of the climate in disease dynamics. It is also important to mention that in reality phytosanitary pod removal will never be $100 \%$ effective. It is time consuming, labor intensive and thus relatively expensive and, in general, only a certain percentage of diseased pods is removed completely, such that inoculum remains in the system capable of causing more infections. Indeed, when pods are eliminated from the trees they are most often left on the ground where they, temporarily, can still cause secondary infections or contribute to the environmental reservoir of spores $\left(S_{p e}\right)$. However, $P$. megakarya will lose "viability" quicker on pods laying on the ground than on pods remaining on the tree. In this paper, the relative importance of the two different inoculum sources $S_{p e}$ and $S_{p i}$ was also investigated. According to the results presented here it also seems that the environmental reservoir is preponderant and seems to play an important role in disease epidemics. Although Turner already mentioned in 1965 that the importance of the soil as a reservoir of inoculum responsible for infections has not been adequately addressed in consideration with Phytophthora cacao pod rot epidemics [38], since then, 
very little work specifically focused on the role and control of on the environmental spore reservoir has been done. This can be explained by difficulties to put in place reliable experiments to estimate some parameters linked to the environmental spore reservoir (Inoculum quantity in the soil and spores shedding or decay rates in the environment). However, recent advances, e.g. the whole genome sequencing of $P$. megakarya, opens up new avenues such as the development of qPCR primers to easily quantify inoculum quantities in the soil [24]. Here we highlight the fact that disease outbreaks and spores lasting in the environment are strongly linked to the environmental spores compartment $S_{p e}$. These results are in agreement with [37] who hypothesize that primary inoculum $\left(S_{p e}\right)$ is the main determinant for the spatial and temporal development of an epidemic at the plantation level and that secondary inoculum $\left(S_{p i}\right)$ is mainly responsible for the within-tree temporal development of black pod. This could also explain why phytosanitation, seems to have a more direct effect on secondary inoculum. This also indicates the need to focus and engage new experimentations to broaden our knowledge on the role of the environmental spore reservoir in disease epidemics in order to establish efficient control strategies. Since the sensitivity analyses also show that the infectious compartments $\left(I, S_{p e}, S_{p i}\right)$ are most sensitive to the Spores inactivation rates parameters $\left(d_{1}\right.$ and $\left.d_{2}\right)$, a more precise estimation of these parameters is needed to confirm these results. In addition to sanitary harvesting, our study also highlights important parameters on which we could act to establish efficient management strategies. For example recommendations in terms of intelligent fungicide applications could lead to a reduction in the production rate of spores, $\sigma$, and treatment of the soil reservoir could lead to increase the inactivation speed of primary and secondary inoculum, i.e. increase the spores death rates $d_{i}$. These strategies combined with intensive and regular sanitary pod removal could guarantee increased yield. This modeling exercise helps us to define and engage new experimentations on the $P$. megakarya-Cocoa pathosystem. It seems especially necessary that we broaden our knowledge on the role of the environmental spore reservoir in disease epidemics and thus establish more efficient management strategies. The next steps will be to develop and implement new experiments to estimate the infectious potential of the environmental reservoir $\left(S_{p, e}\right)$ and how to reduce it. This exercise also shows that for several other parameters used in the model, scarce or no reliable data are available and thus experimentations should be undertaken to provide more reliable estimates for these parameters. Future mathematical studies include the analysis of the impulsive system (11)-(12), and take into account the spatial propagation of the spores, like in [12](see also [14]).

Acknowledgments. The first author is grateful to the DP Agroforesterie Cameroon, the French government and the French Embassy in Yaoundé (SCAC), Cameroon for logistical and financial support during the preparation of this manuscript and also to the CETIC Yaoundé and the CIRAD (UMR AMAP, Montpellier, France) were the manuscript was finalized.

\section{References}

[1] A. Akrofi, I. Amoako-Atta, M. Assuah, And K. Asare, Black pod disease on cacao (Theobroma cacao, l) in ghana: Spread of Phytophthora megakarya and role of economic plants in the disease epidemiology, Crop Protection, 72 (2015), pp. 66-75.

[2] S. Ali, I. Amoako-Attah, R. Bailey, M. Strem, M. Schmidt, A. Akrofi, S. Surujdeo-Maharaj, O. Kolawole, B. Begoude, G. ten Hoopen, E. Goss, W. Phillips-Mora, L. Meinhardt, and B. Bailey, Pcr-based identification of cacao black pod causal agents and identification of biological factors possibly contributing to Phytophthora megakarya's field dominance in west africa, Plant Pathology, 65 (2016), pp. 1095-1108. 
[3] S. Ali, J. Shao, J. David, L. Brent, A. Kronmiller, D. Shen, M. Strem, I. AmoakoAttah, A. Akrofi, B. Begoude, G. ten Hoopen, K. Coulibaly, B. Kebe, R. Melnick, M. Guiltinan, B. Tyler, L. Meinhardt, And B. B.A., Phytophthora megakarya and Phytophthora palmivora, closely related causal agents of cacao black pod rot, underwent increases in genome sizes and gene numbers by different mechanisms, Genome Biol Evol, 9 (2017), pp. 536-537.

[4] J. Avelino, G. Ten Hoopen, And F. DeClerck, Ecological mechanisms for pest and disease control in coffee and cacao agroecosystems of the neotropics. in: Rapidel b, le coq j-f, beer $j$, eds. ecosystem services from agriculture and agroforestry: Measurement and payment, Earthscan Publications, (2011), pp. 91-118.

[5] B. Bailey, S. Ali, A. Akrofi, And M. LW, Phytophthora megakarya, a causal agent of black pod rot in africa.in: Bailey ba, meinhardt lw, editors. cacao diseases, Springer International Publishing, (2016), pp. 267-303.

[6] D. Bainov And P. Simeonov, Systems with impulsive effect: Stability, theory and application, John Wiley \& Sons, 1989.

[7] B. Bani-yaghoub, R. Gautam, Z. Shuai, P. van den Driessche, and P. Ivanek, Reproduction numbers for infections with free living pathogens growing in the environment, Journal of biological dynamics, 6 (2012), pp. 923-940.

[8] A. Berman and R. Plemmons, Nonnegative matrices in the mathematical science, SIAM Philadelphia, (1994).

[9] D. Berry AND C. Cilas, Etude genetique de la reaction a la pourriture brune des cabosses chez des cacaoyers (Theobroma cacao. l) issus d'un plan de croisements diallele, Agronomie, 14 (1994), pp. 638-670.

[10] D. Bisselua, Yede, And S. Vidal, Dispersion models and sampling of cacao mirid bug Sahlbergella singularis (hemiptera: Miridae) on theobroma cacao in southern cameroon, Environmental Entomology, 40 (2011), pp. 111-119.

[11] C. Brasier, M. Griffin, M. Ward, O. Idowu, B. Taylor, and S. Adedoyin, Epidemiology of Phytophthora on cocoa in nigeria final report of the international cocoa black pod research project, Phytopathological Papers, (1981), p. 188.

[12] J. Burie, A. Calonnec, and M. Langlais, Modeling of the Invasion of a Fungal Disease over a Vineyard, Birkhäuser Boston, Boston, MA, 2008, pp. 11-21.

[13] E. J. Butler, Report of the imperial mycologist, Scientific Report of the Research Institute Pusa, (1919), p. 82.

[14] M. Chapwanya and Y. Dumont, On crop vector-borne diseases. impact of virus lifespan and contact rate on the traveling-wave speed of infective fronts, Ecological Complexity, (2017).

[15] J. Cushing and O. Diekmann, The many guises of r0 (a didactic note), Journal of Theoretical Biology, 404 (2016), pp. 295 - 302.

[16] P. Deberdt, C. Mfegue, P. Tondje, M. Bon, M. Ducamp, C. Hurard, B. Begoude, M. Ndoumbe-Nkeng, P. Hebbar, and C. Cilas, Impact of environmental factors, chemical fungicide and biological control on cacao pod production dynamics and black pod disease (Phytophtora megakarya) in cameroon, Biological Control, 44 (2007), pp. 149-159. 
[17] O. Diekmann, J. A. P. Heesterbeek, and M. G. Roberts, The construction of nextgeneration matrices for compartmental epidemic models, Journal of The Royal Society Interface, 7 (2010), pp. 873-885.

[18] M. Efombagn, J. Marelli, M. Ducamp, C. Cilas, S. Nyasse, and D. Vefonge, Effect of fruiting traits on the field resistance of cocoa (Theobroma cacao l.) clones to Phytophthora megakarya, Journal of Phythopathology, 152 (2004), pp. 557-562.

[19] J. Gockowski, M. Tchatat, J. Dondjang, G. Hietet, and T. Fouda, An empirical analysis of the biodiversity and economic returns to cocoa agroforests in southern cameroon, $\mathrm{J}$ Sustain Forl, 29 (2010), pp. 638-670.

[20] J. GoudriaAn And H. VAn LAAR, Modelling potential crop growth processes, Netherlands:Kluwer Academic Publishers, (2007).

[21] D. Guest, Diverse pathogens with a global impact on cocoa yield, Phytopathology, 97 (2007), pp. 1650-1653.

[22] R. Horn And C. Johnson, Matrix analysis, Cambridge University Press, 6 (1985), p. 361.

[23] ICCO, International Cocoa Organization quarterly Bulletin of Cocoa Statistics, vol. XLIII, International Cocoa Organization., 2016.

[24] A. Ippolito, L. Schena, F. Nigro, V. Ligorio, and T. Yaseen, Real-time detection of Phytophthora nicotianae and P. citrophthora in citrus roots and soil, European Journal of Plant Pathology, 110 (2004), pp. $833--843$.

[25] J. Kamgang And G. Sallet, Computation of threshold conditions for epidemiological models and global stability of the disease-free equilibrium (dfe), Mathematical Bioscience, 213 (2008), pp. $1-12$.

[26] J. Konam And D. Guest, Leaf litter mulch reduces the survival of Phytophthora palmivora under cocoa trees in papua new guinea, Australasian Plant Pathology, 31 (2002), pp. 381-383.

[27] M. Luterbacher And A. Akrofi, The current status and distribution of Phytophthora megakarya in ghana., yamoussoukro, ivory coast., In Proceedings of the XIth International Cocoa Research Conference, (1994), pp. 29-35.

[28] S. Marino, I. Hogue, C. Ray, And D. Kirschner, A methodology for performing global uncertainty and sensitivity analysis in systems biology, Journal of Theoretical Biology, 254 (2008), pp. 178-196.

[29] J. Mbarga, B. Begoude, Z. Ambang, M. Meboma, J. Kuate, B. Schiffers, W. EwBAnk, L. Dedieu, and G. Ten Hoopen, A new oil-based formulation of Trichoderma asperellum for the biological control of cacao black pod disease caused by Phytophthora megakarya, Biological Control, 77 (2014), p. 1522.

[30] M. Ndoumbe-Nkeng, C. Cilas, S. Nyemb, E abD Nyasse, D. Bieysse, A. , And I. SACHE, Impact of removing diseased pods on cocoa black pod caused by Phytophthora megakarya and on cocoa production in cameroon, Crop protection, 23 (2004), pp. 415-424.

[31] S. Nyasse, M. Efombagn, B. Kebe, M. Tahi, D. Despreaux, and C. Cilas, Integrated management of Phytophthora diseases on cocoa (Theobroma cacao l): Impact of plant breeding on pod rot incidence, Crop Protection, 26 (2007), pp. 40-45. 
[32] R. Ploetz, The impact of diseases on cacao production: a global overview.in: Bailey ba, meinhardt $l w$, editors. cacao diseases, Springer International Publishing, New York, USA, (2016), pp. 33-59.

[33] W. Soberanis, R. Rios, E. Arevalo, L. Zuniga, O. Cabezas, and U. Krauss, Increased frequency of phytosanitary pod removal in cacao (Theobroma cacao.l) increases yield economically in eastern peru, Crop Protection, 18 (1999), pp. 677-685.

[34] E. Takam Soh, P and Ndong Nguema, H. Gwet, and M. Ndoumbe-Nkeng, Smooth estimation of a lifetime distribution with competing risks by using regular interval observations: application to cocoa fruits growth, Appl.Statist, 62 (2013), pp. 741-760.

[35] P. Takam Soh, M. Ndoumbe-Nkeng, I. Sache, E. Ndong Nguema, H. Gwet, and J. Chadoeuf, Development stage-dependent susceptibility of cocoa fruit to pod rot caused by Phytophthora megakarya, Eur J Plant Pathol, 135 (2013), pp. 363-370.

[36] G. Ten Hoopen, P. Deberdt, M. Mbenoum, and C. Cilas, Modelling cacao pod growth: implications for disease control, Annals of Applied Biology, 160 (2012), pp. 260-272.

[37] G. Ten Hoopen, O. Sounigo, R. Babin, Yede, G. Dikwe, and C. Cilas, Spatial and temporal analysis of a Phytophthora Megakarya epidemic in a plantation in the centre of cameroon, In : 16th International Cocoa Research Conference. Proceedings: towards rational cocoa production and efficient use of a sustainable world cocoa economy, (2012).

[38] P. Turner, Behaviour of Phytophthora palmivora in soil, Plant Disease Reporter, 49 (1965), pp. $135-137$.

[39] P. VAn DEn Driessche, Reproduction numbers of infectious disease models, Infectious Disease Modelling, 2 (2017), pp. 288 - 303.

[40] P. van den Driessche and J. Watmough, Reproduction number and sub-threshold endemic equilibria for compartmental models of disease transmission, Math.Bioscience, 180 (2002), pp. 29-48.

[41] R. VARga, Matrix Iterative Analysis, 1962.

[42] W. Wang AND X.-Q. ZhaO, Threshold dynamics for compartemental epidemic models in periodic environnements, J Dyn Differ Equ, 20 (2008), pp. 699-717. 


\section{Appendix A}

In this appendix we recall some useful results.

Theorem 2 ([25]). Let $\Omega \subset \mathcal{U}=\mathbb{R}_{+}^{3} \times \mathbb{R}_{+}^{3}$. The system $(E)$ is of class $C^{1}$, defined on $\mathcal{U}$. If

(1) $\Omega$ is positively invariant relative to $(E)$.

(2) The system $\dot{x}_{S}=A_{1}(x)\left(x_{S}-x_{D F E, S}\right)$ is GAS at $x_{D F E, S}$.

(3) For any $x \in \Omega$, the matrix $A_{2}(x)$ is metzler irreducible.

(4) There exists a matrix $\bar{A}_{2}$, which is an upper bound of the set $\mathcal{M}=\bar{A}_{2}(x) \in \mathcal{M}_{3}(\mathbb{R}) \mid x \in \bar{\Omega}$, with the property that if $\bar{A}_{2} \in \mathcal{M}$, for any $\bar{x} \in \bar{\Omega}$, such that $A_{2}(\bar{x})=\bar{A}_{2}$, then $\bar{x} \in \Omega$.

(5) The stability modulus of $\bar{A}_{2}, \alpha\left(\bar{A}_{2}\right)=\max _{\lambda \in S p\left(A_{2}\right)} \mathcal{R} e(\lambda)$, satisfies $\alpha\left(\bar{A}_{2}\right) \leq 0$.

Then the DFE is GAS in $\bar{\Omega}$.

Theorem 3 ([22], theorem 6.23). Let $A \in \mathcal{M}_{n}$ be a real matrix. A is irreducible if and only if the matrix $\left(I_{n}+|A|\right)^{n-1}$ is strictly positive.

To check condition (5), following Varga [41] and Berman and Plemmons [8] let us recall the following definition and theorem.

Definition 1 ([41]). For a real Metzler matrix $M, M=N+K$ is a regular splitting if $K$ is a Metzler stable matrix and $N \geq 0$ is a nonnegative matrix .

Theorem 4 ( [41], Theorem 3.29). Let $M=N+K$ be a regular splitting of a real Metzler matrix $M$, then $M$ is Metzler stable if and only if

$$
\rho\left(-N^{-1} K\right)<1
$$

For epidemiological models with periodic parameters, Wang et al. [42] generalized the results from Van den Driessche et al. [40]. Like in [40], it is assumed that all compartments are divided in two types: infected compartments, labeled by $i=1, \ldots, m$, and uninfected compartments, labeled by $i=m+1, \ldots, n$. We consider the following disease transmission system

$$
\frac{d x_{i}}{d t}=f_{i}(x, t)=\mathcal{F}_{i}(x, t)-\left(\mathcal{V}_{i}^{-}(x, t)-\mathcal{V}_{i}^{+}(x, t)\right), \quad i=1, \ldots, n
$$

Let $X_{s}=\left\{x \geq 0, x_{i}=0, i=1, \ldots, m\right\}$. Then, we verify the following assumptions

(A1) For each $1 \leq i \leq n, \mathcal{F}_{i}(x, t), \mathcal{V}_{i}^{+}(x, t)$, and $\mathcal{V}_{i}^{-}(x, t)$ are nonnegative and continuous on $\mathbb{R}_{+}^{n} \times \mathbb{R}_{+}$, and continuously differentiable.

(A2) There is a real number $T>0$ such that $\mathcal{F}_{i}(x, t), \mathcal{V}_{i}^{+}(x, t)$, and $\mathcal{V}_{i}^{-}(x, t)$ are $\tau$-periodic in $t$.

(A3) If $x_{i}=0$, then $\mathcal{V}_{i}^{-}=0$. In particular if $x \in X_{s}$ then $\mathcal{V}_{i}^{-}=0$ for $i=1, \ldots, m$.

(A4) $\mathcal{F}_{i}=0$ for $i>m$.

(A5) If $x \in X_{s}$, then $\mathcal{F}_{i}(x)=\mathcal{V}_{i}^{+}(x)$ for $i=1, \ldots, m$. 
Assume now that system (13) has a periodic equilibrium, $x_{p e r}=\left(0, \ldots, 0, x_{m+1}^{p}, \ldots, x_{n}^{p}\right)$. Then, we compute the Jacobian matrix

$$
D_{x} \mathcal{F}\left(D F E_{1}\right)=\left(\begin{array}{cc}
F(t) & 0 \\
0 & 0
\end{array}\right) \text { and } D_{x} \mathcal{V}\left(D F E_{1}\right)=\left(\begin{array}{cc}
V(t) & 0 \\
J(t) & -M(t)
\end{array}\right)
$$

with

$$
\begin{gathered}
M(t)=\left(\frac{\partial f_{i}\left(x_{p e r}, t\right.}{\partial x_{j}}\right)_{m+1 \leq i, j \leq n} \\
F(t)=\left(\frac{\partial \mathcal{F}_{i}\left(x_{p e r}, t\right.}{\partial x_{j}}\right)_{1 \leq i, j \leq m}, \quad V(t)=\left(\frac{\partial \mathcal{V}_{i}\left(x_{p e r}, t\right.}{\partial x_{j}}\right)_{1 \leq i, j \leq m}
\end{gathered}
$$

Let $\Phi_{M}(t)$ be the monodrony matrix of the linear $\tau$-periodic system $\frac{d z}{d t}=M(t) z$. We assume that $x_{p e r}$ is linearly asymptotically stable in $X_{s}$, that is

(A6) $\rho\left(\Phi_{M}(\tau)\right)<1$.

and finally, the following condition needs to be meet

(A7) $\rho\left(\Phi_{-V}(\tau)\right)<1$.

Thus, if system (13) verifies assumptions (A1)-(A7), then we have

Proposition 4. [42]

- $\mathcal{R}_{0, p e r}=1$ if and only if $\rho\left(\Phi_{F-V}(T)\right)=1$.

- $\mathcal{R}_{0, p e r}<1$ if and only if $\rho\left(\Phi_{F-V}(T)\right)<1$.

- $\mathcal{R}_{0, p e r}>1$ if and only if $\rho\left(\Phi_{F-V}(T)\right)>1$.

Thus, the PDFE is asymptotically stable if $\mathcal{R}_{0, p e r}<1$, and unstable if $\mathcal{R}_{0, p e r}>1$.

In general $\mathcal{R}_{0, p e r}$ is very difficult or impossible to derive analytically. But, following Theorem 2.1 [42], we are able to obtain a numerical approximation. Indeed it suffices to estimate the Floquet multipliers and hence the spectral radius, $\rho(w, \tau)$, of the following linear $\tau$-periodic system

$$
\frac{d w}{d t}=\left(-V(t)+\frac{F(t)}{\lambda}\right) w,
$$

with parameter $\lambda \in(0, \infty)$. Let $W(t, \lambda)$ be the standard fundamental matrix of $(14)$ with $W(0, \lambda)=$ $I d$. Then, according to Theorem 2.1[42] (ii), we know that if $\mathcal{R}_{0, p e r}>0$, then $\lambda=\mathcal{R}_{0, p e r}$ is the unique solution of $\rho(w, \tau)=1$.

\section{Appendix B}

We prove Theorem 1 using Theorem 2, page 27, in Appendix A. Let us now verify the different assumptions:

1. Assumptions (1) and (2) of theorem 2 are satisfied. $\Omega$ is positively invariant as shown in section 3. A direct computation shows that the eigenvalues of $A_{1}(x)$ are real and negative $\left(\lambda_{1, A_{1}}=-\left(\theta_{1}+\mu_{1}+w\right)\right.$, and $\lambda_{2, A_{1}}=-\mu_{2}$, thus the system $\dot{x}_{S}=A_{1}(x)\left(x_{S}-x_{D F E, S}\right)$ is globally asymptotically stable (GAS) at $x_{D F E, S}$. 
2. To verify assumption (3), we use Theorem 3. Clearly $A_{2}(x)$ is a Metzler matrix. Since $n=3$, we have to compute $\left(I_{3}+\left|A_{2}(x)\right|\right)^{2}$, which leads to

$$
\left(I_{3}+\left|A_{2}(x)\right|\right)^{2}=\left(\begin{array}{ccc}
1+\left(\gamma_{i}+\mu_{i}\right) & \beta_{2}^{*} & \beta_{1}^{*} \\
r_{2} \sigma & 1+d_{2} & 0 \\
r_{1} \sigma & 0 & 1+d_{1}
\end{array}\right)\left(\begin{array}{ccc}
1+\left(\gamma_{i}+\mu_{i}\right) & \beta_{2}^{*} & \beta_{1}^{*} \\
r_{2} \sigma & 1+d_{2} & 0 \\
r_{1} \sigma & 0 & 1+d_{1}
\end{array}\right)
$$

where

$$
\beta_{1}^{*}=\frac{\beta_{1}^{1} S_{1}+\beta_{1}^{2} S_{2}}{K_{1}} \quad \text { and } \quad \beta_{2}^{*}=\frac{\beta_{2}^{1} S_{1}+\beta_{2}^{2} S_{2}}{K_{2}}
$$

Finally

$$
\left(I_{3}+\left|A_{2}(x)\right|\right)^{2}=\left(\begin{array}{ccc}
\left(1+\left(\gamma_{i}+\mu_{i}\right)\right)^{2}+r_{2} \sigma \beta_{2}^{*}+r_{1} \sigma \beta_{1}^{*} & \left(2+\gamma_{i}+\mu_{i}+d_{2}\right) \beta_{2}^{*} & \left(2+\gamma_{i}+\mu_{i}+d_{1}\right) \beta_{1}^{*} \\
r_{2} \sigma\left(2+\gamma_{i}+\mu_{i}+d_{2}\right) & r_{2} \sigma \beta_{2}^{*}+\left(1+d_{2}\right)^{2} & r_{2} \sigma \beta_{1}^{*} \\
r_{1} \sigma\left(2+\gamma_{i}+\mu_{i}+d_{1}\right) & r_{1} \sigma \beta_{2}^{*} & r_{1} \sigma \beta_{1}^{*}+\left(1+d_{1}\right)^{2}
\end{array}\right)
$$

which obviously implies $\left(I_{3}+\left|A_{2}(x)\right|\right)^{2}>0$ for all $x \in \Omega /\{(0,0,0,0,0)\}$.

3. Assumption (4): we have to find an upper bound of matrix $A_{2}(x)$. In fact, since $x \in \Omega$, $\bar{A}_{2}$ is simply given by $A_{2}(\bar{x})$, where $\bar{x}=\left(S_{1}^{d f e}, S_{2}^{d f e}, 0,0,0\right) \in \Omega$, with $S_{1}^{d f e}=\frac{\Lambda}{\theta_{1}+\mu_{1}+w}$ and $S_{2}^{d f e}=\frac{\theta_{1} \Lambda}{\mu_{2}\left(\theta_{1}+\mu_{1}+w\right)}$.

4. To check the last assumption of Theorem 2, we first use Definition 1 and Theorem 4 . We consider the following regular splitting of matrix $\bar{A}_{2}$ :

$$
\bar{A}_{2}=\left(\begin{array}{ccc}
-\left(\gamma_{i}+\mu_{i}\right) & 0 & 0 \\
r_{2} \sigma & -d_{2} & 0 \\
0 & 0 & -d_{1}
\end{array}\right)+\left(\begin{array}{ccc}
0 & \frac{\beta_{2}^{1} S_{1}^{d f e}+\beta_{2}^{2} S_{2}^{d f e}}{K_{2}} & \frac{\beta_{1}^{1} S_{1}^{d f e}+\beta_{1}^{2} S_{2}^{d f e}}{K_{1}} \\
0 & 0 & 0 \\
r_{1} \sigma & 0 & 0
\end{array}\right)=N+M
$$

where $N$ is a Metzler stable matrix and $M$ is a non negative matrix. Note carefully that the choice of $N$ and $M$ is fully in agreement with the Jacobian $F$ and $V$ in section 3.1.1

$$
-N^{-1} M=\left(\begin{array}{ccc}
\frac{1}{\left(\gamma_{i}+\mu_{i}\right)} & 0 & 0 \\
\frac{r_{2} \sigma}{\left(\gamma_{i}+\mu_{i}\right) d_{2}} & \frac{1}{d_{2}} & 0 \\
0 & 0 & \frac{1}{d_{1}}
\end{array}\right)\left(\begin{array}{ccc}
0 & \frac{\beta_{2}^{1} S_{1}^{d f e}+\beta_{2}^{2} S_{2}^{d f e}}{K_{2}} & \frac{\beta_{1}^{1} S_{1}^{d f e}+\beta_{1}^{2} S_{2}^{d f e}}{K_{1}} \\
0 & 0 & 0 \\
r_{1} \sigma & 0 & 0
\end{array}\right)
$$




$$
=\left(\begin{array}{ccc}
0 & \frac{\beta_{2}^{1} S_{1}^{d f e}+\beta_{2}^{2} S_{2}^{d f e}}{K_{2}\left(\gamma_{i}+\mu_{i}\right)} & \frac{\beta_{1}^{1} S_{1}^{d f e}+\beta_{1}^{2} S_{2}^{d f e}}{K_{1}\left(\gamma_{i}+\mu_{i}\right)} \\
0 & \frac{r_{2} \sigma}{\left(\gamma_{i}+\mu_{i}\right) d_{2}} \frac{\beta_{2}^{1} S_{1}^{d f e}+\beta_{2}^{2} S_{2}^{d f e}}{K_{2}} & \frac{r_{2} \sigma}{\left(\gamma_{i}+\mu_{i}\right) d_{2}} \frac{\beta_{1}^{1} S_{1}^{d f e}+\beta_{1}^{2} S_{2}^{d f e}}{K_{1}} \\
\frac{r_{1} \sigma}{d_{1}} & 0 & 0
\end{array}\right) .
$$

Now we have to estimate the eigenvalues of $-N^{-1} K$ by computing the characteristic polynomial:

$$
P(X)=\operatorname{det}\left(-N^{-1} M-X I\right)=\left|\begin{array}{ccc}
-X & \frac{\beta_{2}^{1} S_{1}^{d f e}+\beta_{2}^{2} S_{2}^{d f e}}{K_{2}\left(\gamma_{i}+\mu_{i}\right)} & \frac{\beta_{1}^{1} S_{1}^{d f e}+\beta_{1}^{2} S_{2}^{d f e}}{K_{1}\left(\gamma_{i}+\mu_{i}\right)} \\
0 & \frac{r_{2} \sigma}{\left(\gamma_{i}+\mu_{i}\right) d_{2}} \frac{\beta_{2}^{1} S_{1}^{d f e}+\beta_{2}^{2} S_{2}^{d f e}}{K_{2}}-X & \frac{r_{2} \sigma}{\left(\gamma_{i}+\mu_{i}\right) d_{2}} \frac{\beta_{1}^{1} S_{1}^{d f e}+\beta_{1}^{2} S_{2}^{d f e}}{K_{1}} \\
\frac{r_{1} \sigma}{d_{1}} & 0 & -X
\end{array}\right|
$$

which leads to

$$
P(X)=X^{2}\left[\frac{r_{2} \sigma}{\left(\gamma_{i}+\mu_{i}\right) d_{2}} \frac{\beta_{2}^{1} S_{1}^{d f e}+\beta_{2}^{2} S_{2}^{d f e}}{K_{2}}-X\right]+\frac{r_{1} \sigma}{d_{1}} \frac{\beta_{1}^{1} S_{1}^{d f e}+\beta_{1}^{2} S_{2}^{d f e}}{K_{1}\left(\gamma_{i}+\mu_{i}\right)} X .
$$

According to (6), we deduce

$$
P(x)=X\left(\mathcal{R}_{0, i}+\mathcal{R}_{0, d} X-X^{2}\right) .
$$

which implies that

$$
\rho\left(-N^{-1} M\right)=\frac{1}{2}\left(\mathcal{R}_{0, d}+\sqrt{\mathcal{R}_{0, d}^{2}+4 \mathcal{R}_{0, i}}\right)=\mathcal{R}_{0} .
$$

Then according to theorem 4 , page 27 , we conclude that $\alpha\left(\bar{A}_{2}\right) \leq 0$ if and only if $\mathcal{R}_{0} \leq 1$.

Altogether, all assumptions of Theorem 2, page 27, being verified, we deduce that the DFE is GAS if $\mathcal{R}_{0} \leq 1$.

\section{Appendix C: Computations of the Endemic Equilibrium}

Replacing $S_{1}^{*}, S_{2}^{*}, S_{p, i}$, and $S_{p, e}$ by their respective expressions in $(1)_{4}$, we show that the endemic equilibrium of $(S)$, satisfy a polynomial of the form

$$
P(I)=a_{4} I^{4}+a_{3} I^{3}+a_{2} I^{2}+a_{1} I+a_{0} .
$$

In the following, we compute the different terms of $P(I)$ using the expression of $S_{1}$ and $S_{2}$ at the equilibrium:

$$
S_{1}=\frac{\left(\theta_{1}+\mu_{1}+w\right) S_{1}^{d f e}}{\theta_{1}+\mu_{1}+w+\lambda_{1}}, \quad S_{2}=\frac{\left(\mu_{2}+\theta_{2}\right) S_{2}^{d f e}}{\mu_{2}+\theta_{2}+\lambda_{2}}
$$




$$
\left\{\begin{aligned}
S_{1}^{d f e} & =\frac{\Lambda}{\theta_{1}+\mu_{1}+w} \\
S_{2}^{d f e} & =\frac{\theta_{1} \Lambda}{\left(\mu_{2}+\theta_{2}\right)\left(\theta_{1}+\mu_{1}+w\right)}
\end{aligned}\right.
$$

Replacing $S_{p, e}$ and $S_{p, i}$ in the infection forces we get:

$$
\lambda_{i}=\left[\frac{\beta_{1}^{i} r_{1} \sigma I}{K_{1} d_{1}+r_{1} \sigma I}+\frac{\beta_{2}^{i} r_{2} \sigma I}{K_{2} d_{2}+r_{2} \sigma I}\right]=\frac{\left[\left(\beta_{1}^{i}+\beta_{2}^{i}\right) r_{1} r_{2} \sigma^{2}\right] I^{2}+\left[\beta_{1}^{i} r_{1} \sigma K_{2} d_{2}+\beta_{2}^{i} r_{2} \sigma K_{1} d_{1}\right] I}{r_{1} r_{2} \sigma^{2} I^{2}+\left[r_{1} \sigma K_{2} d_{2}+r_{2} \sigma K_{1} d_{1}\right] I+K_{1} K_{2} d_{1} d_{2}}
$$

Then, using $(5)_{3}$, we have:

$$
\begin{aligned}
\left(\gamma_{I}+\mu_{I}\right) I & =\lambda_{1} S_{1}+\lambda_{2} S_{2} \\
& =\frac{\left(A_{2}^{1} I^{2}+A_{1}^{1} I\right)\left(\theta_{1}+\mu_{1}\right) S_{1}^{d f e}}{\left(B_{2} I^{2}+B_{1} I+B_{0}\right)\left(\theta_{1}+\mu_{1}+\lambda_{1}\right)}+\frac{\left(A_{2}^{2} I^{2}+A_{1}^{2} I\right) \mu_{2} S_{2}^{d f e}}{\left(B_{2} I^{2}+B_{1} I+B_{0}\right)\left(\mu_{2}+\lambda_{2}\right)} \\
& =\frac{\left[A_{2}^{1} S_{1}^{d f e}\right] I^{2}+\left[A_{1}^{1} S_{1}^{d f e}\right] I}{\left[B_{2}+\frac{A_{2}^{1}}{\theta_{1}+\mu_{1}}\right] I^{2}+\left[B_{1}+\frac{A_{1}^{1}}{\theta_{1}+\mu_{1}}\right] I+B_{0}}+\frac{\left[A_{2}^{2} S_{2}^{d f e}\right] I^{2}+\left[A_{1}^{2} S_{2}^{d f e}\right] I}{\left[B_{2}+\frac{A_{2}^{2}}{\mu_{2}}\right] I^{2}+\left[B_{1}+\frac{A_{1}^{2}}{\mu_{2}}\right] I+B_{0}},
\end{aligned}
$$

or equivalently

$$
\left(\gamma_{I}+\mu_{I}\right) I=\frac{F_{1} I^{2}+F_{2} I}{F_{3} I^{2}+F_{4} I+B_{0}}+\frac{P_{1} I^{2}+P_{2} I}{P_{3} I^{2}+P_{4} I+B_{0}}
$$

with

$$
\begin{aligned}
& F_{1}=\left[\left(\beta_{1}^{1}+\beta_{2}^{1}\right) r_{1} r_{2} \sigma^{2}\right] S_{1}^{d f e}, \\
& P_{1}=\left[\left(\beta_{1}^{2}+\beta_{2}^{2}\right) r_{1} r_{2} \sigma^{2}\right] S_{2}^{d f e}, \\
& F_{2}=\left[\beta_{1}^{1} r_{1} \sigma K_{2} d_{2}+\beta_{2}^{1} r_{2} \sigma K_{1} d_{1}\right] S_{1}^{d f e}, \\
& P_{2}=\left[\beta_{1}^{2} r_{1} \sigma K_{2} d_{2}+\beta_{2}^{2} r_{2} \sigma K_{1} d_{1}\right] S_{2}^{d f e}, \\
& F_{3}=r_{1} r_{2} \sigma^{2}\left[1+\frac{\left(\beta_{1}^{1}+\beta_{2}^{1}\right)}{\theta_{1}+\mu_{1}}\right], \\
& P_{3}=r_{1} r_{2} \sigma^{2}\left[1+\frac{\left(\beta_{1}^{2}+\beta_{2}^{2}\right)}{\mu_{2}+\theta_{2}}\right], \\
& F_{4}=\left[1+\frac{\beta_{1}^{1}}{\theta_{1}+\mu_{1}}\right] r_{1} \sigma K_{2} d_{2}+\left[1+\frac{\beta_{2}^{1}}{\theta_{1}+\mu_{1}}\right] r_{2} \sigma K_{1} d_{1}, \\
& P_{4}=\left[1+\frac{\beta_{1}^{2}}{\mu_{2}}\right] r_{1} \sigma K_{2} d_{2}+\left[1+\frac{\beta_{2}^{2}}{\mu_{2}}\right] r_{2} \sigma K_{1} d_{1}, \\
& B_{0}=K_{1} K_{2} d_{1} d_{2},
\end{aligned}
$$

from (15), we have

$$
\left(\gamma_{I}+\mu_{I}\right) I=\frac{F_{1} I^{2}+F_{2} I}{F_{3} I^{2}+F_{4} I+B_{0}}+\frac{P_{1} I^{2}+P_{2} I}{P_{3} I^{2}+P_{4} I+B_{0}}
$$

which leads directly to

$$
\left(\gamma_{I}+\mu_{I}\right) I=\frac{A I^{4}+B I^{3}+C I^{2}+D I}{E I^{4}+F I^{3}+G I^{2}+H I+B_{0}^{2}}
$$

where

$$
\left\{\begin{array}{l}
A=F_{1} P_{3}+P_{1} F_{3} \\
B=F_{1} P_{4}+P_{1} F_{4}+F_{2} P_{3}+P_{2} F_{3} \\
C=B_{0}\left(F_{1}+P_{1}\right)+F_{2} P_{4}+P_{2} F_{4} \\
D=B_{0}\left(F_{2}+P_{2}\right) \\
E=F_{3} P_{3} \\
F=F_{3} P_{4}+P_{3} F_{4} \\
G=B_{0}\left(F_{3}+P_{3}\right)+F_{4} P_{4} \\
H=B_{0}\left(F_{4}+P_{4}\right)
\end{array}\right.
$$


Then, equation (16) leads directly to:

$$
\begin{aligned}
P(I)= & \left(\gamma_{I}+\mu_{I}\right) E * I^{4}+\left[\left(\gamma_{I}+\mu_{I}\right) F-A\right] * I^{3}+\left[\left(\gamma_{I}+\mu_{I}\right) G-B\right] * I^{2}+ \\
& +\left[\left(\gamma_{I}+\mu_{I}\right) H-C\right] * I+\left[\left(\gamma_{I}+\mu_{I}\right) B_{0}^{2}-D\right],
\end{aligned}
$$

which is equivalent to

$$
P(I)=a_{4} I^{4}+a_{3} I^{3}+a_{2} I^{2}+a_{1} I+a_{0},
$$

with 
Table 1: Epidemiological parameters

\begin{tabular}{|c|c|c|c|c|}
\hline Parameters & description & Units & Values or range of values & Source \\
\hline$\Lambda$ & Cherelle "recruitment" rate & Days $^{-1}$ & 12 & {$[10]$} \\
\hline$\theta_{1}$ & $\begin{array}{c}\text { Growth rate from cherelle } \\
\text { to young pod }\end{array}$ & Days $^{-1}$ & 0.05 & {$[36][34]$} \\
\hline$\theta_{2}$ & ripening rate & Days $^{-1}$ & 0.027 & \\
\hline$\beta_{1}^{1}$ & $\begin{array}{l}\text { primary infection rate at } \\
\text { the cherelle stage }\end{array}$ & Days $^{-1}$ & 0.05 & Assumed \\
\hline$\beta_{2}^{1}$ & $\begin{array}{c}\text { secondary infection rate at } \\
\text { the cherelle stage }\end{array}$ & Days $^{-1}$ & 0.05 & Assumed \\
\hline$\beta_{1}^{2}$ & $\begin{array}{c}\text { primary infection rate at } \\
\text { the pod stage }\end{array}$ & Days $^{-1}$ & 0.2 & Assumed \\
\hline$\beta_{2}^{2}$ & $\begin{array}{c}\text { secondary infection rate at } \\
\text { the pod stage }\end{array}$ & Days $^{-1}$ & 0.2 & Assumed \\
\hline$\gamma_{I}$ & $\begin{array}{c}\text { rate of phytosanitary } \\
\text { pod removal }\end{array}$ & Days $^{-1}$ & {$[0-0.8]$} & {$[30]$} \\
\hline$w$ & attack rate by wilt & Days $^{-1}$ & 0.1 & {$[35]$} \\
\hline$\mu_{1}$ & $\begin{array}{c}\text { natural death rate of } \\
\text { cherelle }\end{array}$ & Days $^{-1}$ & 0.05 & {$[34]$} \\
\hline$\mu_{2}$ & natural death rate of pods & Days $^{-1}$ & 0.00469 & {$[34]$} \\
\hline$\mu_{I}$ & $\begin{array}{c}\text { natural death rate of } \\
\text { infected pods }\end{array}$ & Days $^{-1}$ & 0.05 & Assumed \\
\hline$d_{1}$ & $\begin{array}{c}\text { natural decay of spores in } \\
\text { the environment }\end{array}$ & Days $^{-1}$ & 0.02 & Assumed \\
\hline$d_{2}$ & inactivation speed of spores & Days $^{-1}$ & 0.4 & Assumed \\
\hline$\sigma$ & $\begin{array}{l}\text { production rate of spores by } \\
\text { infected pods }\end{array}$ & Spores Days ${ }^{-1}$ & 574200 & Assumed \\
\hline$r_{2}$ & releasing speed of spore & Spores Days ${ }^{-1}$ & 0.4 & Assumed \\
\hline$r_{1}$ & $\begin{array}{l}\text { shedding rate of spores in the } \\
\text { environment }\end{array}$ & Spores Days ${ }^{-1}$ & 0.4 & Assumed \\
\hline$K_{2}$ & $\begin{array}{l}\text { Michaelis constant for } \\
\text { pod to pod transmission }\end{array}$ & Number of spores & $10^{8}$ & Assumed \\
\hline$K_{1}$ & $\begin{array}{l}\text { Michaelis constant for } \\
\text { environmental transmission }\end{array}$ & Number of spores & $2 \times 10^{9}$ & Assumed \\
\hline
\end{tabular}

\begin{tabular}{|c|c|c|}
\hline$\gamma_{I}$ & $\mathcal{R}_{0}$ & $\mathcal{R}_{0, p e r}$ \\
\hline 0 & 3.27 & 3.22 \\
\hline 0.25 & 0.88 & 1.03 \\
\hline 0.3 & 0.80 & 0.93 \\
\hline
\end{tabular}

Table 2: Thresholds estimates for different values of $\gamma_{I}$ when $\Lambda$ constant and $\Lambda$ periodic 


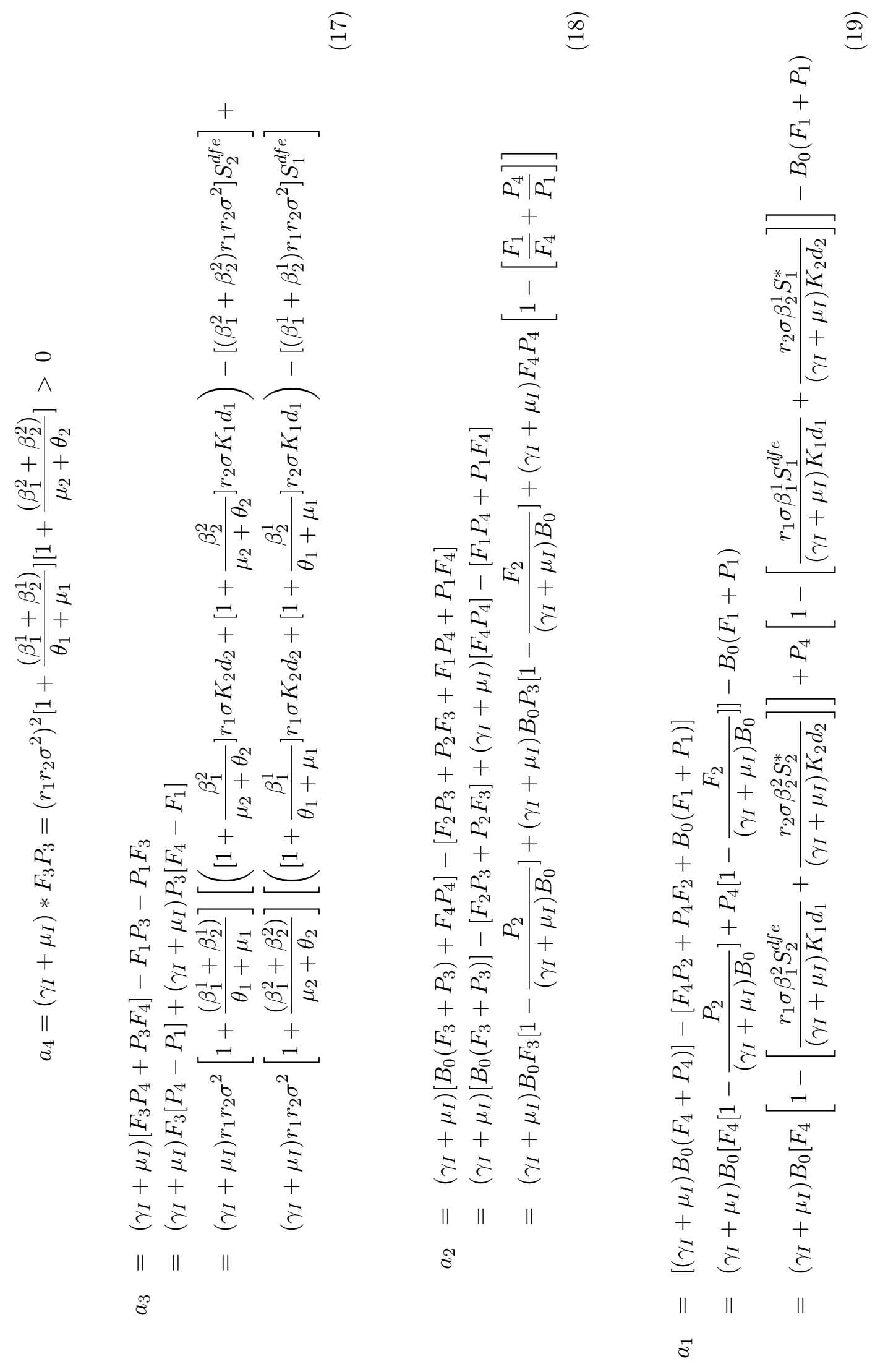




$$
\begin{aligned}
a_{0} & =\left[\left(\gamma_{I}+\mu_{I}\right) B_{0}^{2}-B_{0}\left(F_{2}+P_{2}\right)\right] \\
& \left.=B_{0}^{2}\left[\left(\gamma_{I}+\mu_{I}\right)-\frac{F_{2}+P_{2}}{B_{0}}\right]\right] \\
& =\left(\gamma_{I}+\mu_{I}\right) B_{0}^{2}\left[\left[1-\frac{F_{2}+P_{2}}{\left(\gamma_{I}+\mu_{I}\right) B_{0}}\right]\right. \\
& =\left(\gamma_{I}+\mu_{I}\right) B_{0}^{2}\left[1-\left[\frac{r_{1} \sigma\left(\beta_{1}^{1} S_{1}^{d f e}+\beta_{1}^{2} S_{2}^{d f e}\right)}{\left(\gamma_{I}+\mu_{I}\right) K_{1} d_{1}}+\frac{r_{2} \sigma\left(\beta_{2}^{1} S_{1}^{d f e}+\beta_{2}^{2} S_{2}^{d f e}\right)}{\left(\gamma_{I}+\mu_{I}\right) K_{2} d_{2}}\right]\right] \\
& =\left(\gamma_{I}+\mu_{I}\right) B_{0}^{2}\left[1-\mathcal{R}_{0, g}\right]
\end{aligned}
$$

Obviously, we can deduce that the sign of $a_{0}$ depends on $\mathcal{R}_{0, g}$.

We clearly have an idea of the sign of $a_{0}$, which is directly related to $\mathcal{R}_{0, g}$.

Thus, when $\mathcal{R}_{0, g}>1$ than $a_{0}<0$, which implies that there exists at least one positive root of $P(I)$, and thus, at least, one positive equilibrium.

However, using the Descarte's rule of sign, we can go further and derive the possible number of positive roots of $P(I)$. The different possible results are summarized in Table 3, page 35 .

Table 3: Descartes sign tab for the polynom $P(I)$ when $\mathcal{R}_{0}>1$

\begin{tabular}{|c|c|c|c|c|c|c|c|}
\hline No: & $a_{4}$ & $a_{3}$ & $a_{2}$ & $a_{1}$ & $a_{0}$ & $\begin{array}{c}\text { Number of } \\
\text { signs changes }\end{array}$ & $\begin{array}{c}\text { Number of } \\
\text { positive roots }\end{array}$ \\
\hline 1 & + & + & + & - & - & 1 & 1 \\
\hline 2 & + & + & - & - & - & 1 & 1 \\
\hline 3 & + & - & + & - & - & 3 & 3 or 1 \\
\hline 4 & + & - & - & - & - & 1 & 1 \\
\hline 5 & + & + & + & + & - & 1 & 3 or 1 \\
\hline 6 & + & + & - & + & - & 3 & 3 or 1 \\
\hline 7 & + & - & + & + & - & 3 & 3 or 1 \\
\hline 8 & + & - & - & + & - & 3 & \\
\hline
\end{tabular}


Table 4: Range of values for the parameters used for the global sensitivity analysis

\begin{tabular}{|c|c|}
\hline Parameters & range of values \\
\hline$\Lambda$ & {$\left[\begin{array}{ll}0 & 100\end{array}\right]$} \\
\hline$\theta_{1}$ & {$\left[\begin{array}{ll}0.01 & 0.2\end{array}\right]$} \\
\hline$\theta_{2}$ & {$\left[\begin{array}{ll}0.01 & 0.2\end{array}\right]$} \\
\hline$\beta_{1}^{1}$ & {$\left[\begin{array}{ll}0.01 & 0.5\end{array}\right]$} \\
\hline$\beta_{2}^{1}$ & {$\left[\begin{array}{ll}0.01 & 0.5\end{array}\right]$} \\
\hline$\beta_{1}^{2}$ & {$\left[\begin{array}{ll}0.01 & 0.5\end{array}\right]$} \\
\hline$\beta_{2}^{2}$ & {$\left[\begin{array}{ll}0.01 & 0.5\end{array}\right]$} \\
\hline$\gamma_{I}$ & {$\left[\begin{array}{ll}0 & 0.8\end{array}\right]$} \\
\hline$w$ & {$\left[\begin{array}{ll}0.01 & 0.4\end{array}\right]$} \\
\hline$\mu_{1}$ & {$\left[\begin{array}{ll}0.01 & 0.2\end{array}\right]$} \\
\hline$\mu_{2}$ & {$\left[\begin{array}{ll}0.0001 & 0.1\end{array}\right]$} \\
\hline$\mu_{I}$ & {$\left[\begin{array}{ll}0.01 & 0.2\end{array}\right]$} \\
\hline$d_{1}$ & {$\left[\begin{array}{ll}0.01 & 0.5\end{array}\right]$} \\
\hline$d_{2}$ & {$\left[\begin{array}{ll}0.01 & 0.5\end{array}\right]$} \\
\hline$\sigma$ & {$\left[\begin{array}{ll}10^{3} & 10^{6}\end{array}\right]$} \\
\hline$r_{2}$ & {$\left[\begin{array}{ll}0.01 & 0.8\end{array}\right]$} \\
\hline$r_{1}$ & {$\left[\begin{array}{ll}0.01 & 0.8\end{array}\right]$} \\
\hline$K_{2}$ & {$\left[\begin{array}{ll}0 & 10^{10}\end{array}\right]$} \\
\hline$K_{1}$ & {$\left[\begin{array}{ll}0 & 10^{10}\end{array}\right]$} \\
\hline
\end{tabular}

